\title{
A cytokine receptor-masked IL2 prodrug selectively activates tumor-infiltrating lymphocytes for potent antitumor therapy
}

Eric Hsu

UT Southwestern Medical Center

\section{Xuezhi Cao}

Institut Pasteur of Shanghai

Benjamin Moon

UT Southwestern Medical Center https://orcid.org/0000-0003-4390-3560

joonbeom Bae

UT Southwestern Medical Center

\section{Zhichen Sun}

Institute of Biophysics, Chinese Academy of Sciences https://orcid.org/0000-0001-7837-8407

\section{Zhida Liu}

The University of Texas Southwestern Medical Center https://orcid.org/0000-0002-3980-8440

\section{Yang-Xin Fu ( $\nabla$ yang-xin.fu@utsouthwestern.edu )}

UT Southwestern Medical Center https://orcid.org/0000-0002-4809-825X

\section{Article}

Keywords: Tumor-associated Protease Substrate, CD8 T Cells, Cytokine Storm, Immune Checkpoint Blockade, Abscopal Effect

Posted Date: October 21st, 2020

DOI: https://doi.org/10.21203/rs.3.rs-92075/v1

License: (a) (1) This work is licensed under a Creative Commons Attribution 4.0 International License. Read Full License

Version of Record: A version of this preprint was published at Nature Communications on May 13th, 2021. See the published version at https://doi.org/10.1038/s41467-021-22980-w. 


\section{lymphocytes for potent antitumor therapy}

4 Eric J. Hsu ${ }^{1}$, Xuezhi Cao ${ }^{2 *}$, Benjamin Moon ${ }^{1}$, Joonbeom Bae ${ }^{2}$, Zhichen Sun ${ }^{2,3}$, Zhida Liu ${ }^{2}$ and $5 \quad$ Yang-Xin $\mathrm{Fu}^{1,2, *}$

$7 \quad{ }^{1}$ Department of Immunology, UT Southwestern Medical Center, Dallas, TX 75390, USA

$8 \quad{ }^{2}$ Department of Pathology, UT Southwestern Medical Center, Dallas, TX 75390, USA

$9{ }^{3}$ Department of Biomedical Engineering, UT Southwestern Medical Center, Dallas, TX 75390, 10 USA

11 *Correspondence: Yang-Xin Fu or Xuezhi Cao; UT Southwestern Medical Center, Dallas, TX

12 75390,USA; Yang-Xin.Fu@UTSouthwestern.edu; Xuezhi.Cao@UTSouthwestern.edu

14 Conflict of interest statement

15 The authors have declared that no competing interest exists. 


\section{Summary}

17 Interleukin-2 (IL-2) is an FDA approved treatment for multiple metastatic cancers. However, while 18 IL-2 is a potent activator of CD8 and NK cells, its clinical use is limited by short in vivo half-life,

19 low potency, and severe in vivo toxicity. Current strategies of reducing IL-2 receptor alpha binding 20 and increasing IL-2 receptor beta binding reduce efficacy or increase toxicity. Here, we address 21 these issues by engineering a novel IL-2 prodrug (ProIL2). We mask the activity of a CD8 T cell22 preferred IL-2 mutein/Fc fusion protein with IL2 receptor beta linked to a tumor-associated 23 protease substrate. ProIL2 restores activity after cleavage by tumor-associated enzymes, and 24 preferentially activates inside tumors, where it is efficiently cleaved and expands CD8 T cells. This 25 significantly reduces IL-2 associated cytokine storm, body weight loss, and mortality without 26 compromising antitumor efficacy. ProIL2 also overcomes resistance of cancers to immune 27 checkpoint blockade. Lastly, neoadjuvant ProIL2 treatment can effectively eliminate metastatic 28 cancer by inducing an abscopal effect. Taken together, our approach presents an effective tumor 29 targeting therapy with reduced toxicity. 
33 Interleukin-2 (IL-2) is a potent cytokine that activates cytotoxic T cells and NK cells ${ }^{1}$. Because it 34 so potently induces immune system activation, IL-2 was approved by the FDA as a treatment for 35 renal cell carcinoma and metastatic melanoma ${ }^{2}$. Unfortunately, IL-2 cancer immunotherapy 36 encounters three major issues in the clinic. First, IL-2 does not specifically activate cytotoxic 37 lymphocytes (CTLs) in the tumor and is used up by many IL-2 sinks in the periphery ${ }^{3}$. As a result, 38 it has a short half-life ${ }^{4}$. Second, IL-2 binds with more affinity to the high affinity IL-2 receptor 39 alpha (IL2R $\alpha$ ), which is constitutively expressed on Tregs, than to the lower affinity IL-2 receptor 40 beta (IL2R $\beta$ ), which is expressed on CD8 T cells, Tregs, and NK cells ${ }^{1,5-7}$. Third, in part due to 41 this high affinity for the trimeric high-affinity receptor complex on immunosuppressive Tregs, a 42 higher dose of IL-2 is necessary to achieve CTL mediated immune killing of the tumor, which 43 leads to severe toxicity in most patients ${ }^{2}$.

44 Multiple strategies have been tested to address these three limitations. Synthesizing IL-2/Fc fusion 45 proteins increases IL-2 half-life but increase toxicity. PEGylation of IL-2 can significantly improve 46 IL-2 half-life but generation of consistent site-specific PEGylated IL-2 forms is a challenge to 47 manufacturers. In addition, lack of tumor-targeted release of PEGylated IL-2 results in toxicity 48 before reaching therapeutic doses, which limits repeatable results in clinical trials ${ }^{8,9}$. Other groups 49 have synthesized IL-2 muteins that increase IL-2 binding to IL2R $\beta$ on CD8 T cells ${ }^{10-12}$. Increased 50 IL-2 selectivity of CD8 T cells may reduce its targeting and activation of Tregs but increases 51 overall toxicity. There is an urgent need to target IL-2 binding to effector cells specifically in tumor 52 tissues without causing peripheral toxicity. 
53 In this study, we synthesized a cytokine receptor-masked IL-2 prodrug (ProIL2) that exhibits 54 minimal peripheral cytotoxicity. ProIL2 is cleaved and activated by tumor specific proteases into 55 its free form, which is an IL-2 mutein denoted as SumIL2-Fc. ProIL2 preferentially expands CTLs 56 in the tumor and has equivalent antitumor efficacy so SumIL2-Fc. Matrix metalloproteinases 57 (MMPs) have been observed to play a role in tumor angiogenesis and invasion by digesting cell58 matrix adhesions in cancer tissues, and are thus highly expressed on multiple tumor types ${ }^{13}$. 59 Because they are highly expressed in many tumors and on inflammatory cells, we choose to use 60 MMPs as our target tumor protease ${ }^{14}$. Overall, our effective and safe immunotherapy both presents 61 a novel option for combatting difficult clinical issues in the cancer field and serves as a platform 62 for developing novel potent cytokine and antibody therapies in the future. 
63

64 65 efficacy

66

67 we designed ProIL2 to have four domains: human IgG1, an IL-2 variant, an IL-2 receptor, and a

\section{Results}

Engineering an optimal ProIL2 with high IL-2 blocking activity, MMP cleavage, and antitumor

To allow IL-2 to preferentially target tumor infiltrating lymphocytes (TILs) with long half-life, flexible MMP-cleavable linker. We modulate and engineer each of these four domains to maximize three criteria: blocking of IL-2 activity when uncleaved, MMP cleavability, and antitumor efficacy (Figure 1a). We initially tested a homodimeric format of ProIL2, which, in N to $\mathrm{C}$ terminus order, incorporates IL2R $\beta$, the MMP cleavable linker, and SumIL2 fused onto each "arm" of Fc. Intriguingly, this ProIL2 design exhibited no antitumor efficacy and could not be digested by MMPs, which raised the possibility that MMPs failed to access the cleavable linker (Supplementary Figure 1a). As a result, we engineered designs of ProIL2 to minimize potential steric clash that would prevent MMP access of the cleavable linker. These engineering strategies involved synthesizing hIgG1 heterodimers ${ }^{15}$, incorporating different or truncated IL-2 receptor subunits, and altering the orientation of domains on both the $\mathrm{N}$ and $\mathrm{C}$ termini of each $\mathrm{Fc}$ arm. We initially rejected the designs that exhibited minimal IL-2 blocking activity and recombinant MMP cleavage. For each ProIL2 that passed in vitro screening, we injected MC38 tumor bearing mice with PBS or ProIL2 and quantified the amount of tumor reduction by each ProIL2 treatment (Figure 1b). After determining which design exhibited the best antitumor efficacy, we tested modifications of the Fc region for this version of ProIL2. We observed that ProIL2 using wild-type hIgG1 Fc exhibited the same antitumor efficacy as using a mutant Fc that depletes antibody-dependent cell-mediated cytotoxicity ${ }^{16}$, but with reduced toxicity

(Supplementary Figure 1b-c). Thus, we chose to use wild-type hIgG1 Fc. 
We ultimately determined that the design in Figure 2a maximized our three screening criteria.

87 On one Fc arm, we fused SumIL2 to the N terminus of wild-type Fc, linked together by a

GGGGS linker. On the other Fc arm, we fused the full extracellular domain of IL2R $\beta$ to the N terminus of Fc, linked together by a flexible linker that includes a synthetically determined 10 amino acid MMP protease substrate (Figure 2a). We purified and eluted this ProIL2 using Protein A chromatography and assessed its purity via SDS-PAGE, noting that we indeed created one single band of appropriate approximate size (Supplementary Figure 2a). Purification of this protein was high yield. This protein is also very stable, not degrading and still having antitumor activity even after 3 months of storage at $4^{\circ} \mathrm{C}$. Overall, we tested numerous designs of ProIL2, modifying each domain of ProIL2 until we synthesized the most optimal form of ProIL2. From here on, the phrase "ProIL2" refers to our most optimal prodrug design in Figure 2a.

\section{ProIL2 blocks IL-2 activity when uncleaved}

We tested the ability of IL2R $\beta$ to block both SumIL2 binding and functional activity when ProIL2 was uncleaved. First, we used flow cytometry to stain for ProIL2 binding to the surface of cells that express IL-2 receptor subunits. Compared to SumIL2-Fc $\left(\mathrm{IC}_{50}=1.1 \pm 0.2 \mathrm{nM}\right)$, ProIL2 $\left(\mathrm{IC}_{50}=14.4 \pm 1.4 \mathrm{nM}\right)$ bound with 13 -fold lower affinity to these cells (Figure $2 \mathrm{~b}$ ). We also used an HEK-Blue ${ }^{\mathrm{TM}}$ IL-2 reporter cell assay to compare functional activity of these two proteins. Similarly to the binding assay, ProIL2 $\left(\mathrm{IC}_{50}=371.6 \pm 50.0 \mathrm{pM}\right)$ also induced 8.5 -fold less activity than SumIL2-Fc $\left(\mathrm{IC}_{50}=43.9 \pm 4.5 \mathrm{pM}\right)$ (Figure $\left.2 \mathrm{c}\right)$.

We also assessed whether ProIL2 could cleave efficiently in vitro. We incubated ProIL2 with recombinant MMPs for multiple time points and then quantified the amounts of uncleaved and cleaved ProIL2 via SDS-PAGE (Supplementary Figure 2a). MMPs are able to cleave ProIL2 
108

109

110

111 112 vitro.

gradually over time, cleaving $70 \%$ of the initial amount of ProIL 2 over 4 hours of incubation (Figure 2d). ProIL2 that has been sufficiently cleaved also has activity close to SumIL2-Fc, as demonstrated by IL-2 reporter cell assay (Figure 2e). Taken together, our design of ProIL2 effectively blocks SumIL2-Fc binding and stimulatory activity and is avidly cleaved by MMPs in

ProIL2 preferentially localizes to tumors and is cleaved intratumorally in vivo

114 Because ProIL2 cleaved well in response to MMPs, we assessed whether ProIL2 could also be 115 cleaved in vivo, preferentially in the tumor. We first quantified the serum half-life of ProIL2. 116 After injecting ProIL2 intraperitoneally into tumor bearing mice, we calculated its serum half117 life to be 15.1 hours (Figure 3a). This is much longer than that of recombinant IL-2 $(<15$ 118 minutes) and comparable to the 14.6 hours of SumIL2-Fc ${ }^{17,18}$.

119 To determine how ProIL2 distributed into and cleaved in different tissues, we injected ProIL2 120 into CT26 tumor bearing BALB/c mice and extracted and homogenized tumors, livers, spleens, 121 and lungs 24 hours after injection. We first quantified the amount of ProIL2 in each tissue

122 homogenate. 2-4 times more ProIL2 per total amount of tissue localized into tumors compared to 123 all of the other tissues (Figure 3b). We also determined the levels of multiple inflammatory 124 cytokines in each tissue in response to ProIL2. Tumor tissue showed a high amount of IFN $\gamma$ and 125 TNF $\alpha$ (Figure 3c, Supplementary Figure 2b), which is consistent with a phenotype of TIL 126 activation $^{19-21}$.

127 We also aimed to quantify whether and how much ProIL2 would cleave in tumors in vivo. We 128 used Protein A immunoprecipitation and Western Blot Analysis to quantify the amount and ratio 129 of uncleaved and cleaved ProIL2 in each extracted tissue homogenate (Supplementary Figure 
130 2c). On average, over 50\% of ProIL2 in the tumor was cleaved, compared to less than $25 \%$ in the 131 liver, lung, and spleen (Figure 3d). We also compared the amount of cleaved ProIL2 between 132 tissues, and observed that cleaved ProIL2 product was staggeringly most prevalent in the tumor 133 compared to any other tissue (Figure 3e). Similar results were observed in a B16 tumor bearing 134 mouse model (Supplementary Figure 3). Altogether, these results demonstrate that ProIL2 135 preferentially localizes to and cleaves in tumors but not non-tumor tissues.

ProIL2 exhibits reduced toxicity compared to SumIL2-Fc without compromising anti-tumor

137 efficacy

138 We have emphasized the necessity of reducing peripheral toxicity with our prodrug. However, its 139 anti-tumor efficacy and ability to expand TILs should be comparable to that of SumIL2-Fc. We 140 intraperitoneally injected equimolar doses of ProIL2 or SumIL2-Fc into MC38 tumor bearing 141 mice and observed that while ProIL2 induced significantly less body weight loss compared to 142 SumIL2-Fc, the two treatments exhibited equivalent efficacy in causing tumor regression (Figure 143 4a-b, Supplementary Figure 4a). At our lowest observed dose of maximum antitumor efficacy, 144 we consistently observed this phenomenon in multiple tumor models (Supplementary Figure 4b$145 \mathrm{e})$.

146 We also examined cytokine related toxicity of ProIL2. We treated tumor bearing mice with PBS, 147 SumIL2-Fc, or ProIL2, collected serum from these mice 24 hours post treatment, and quantified 148 the level of serum inflammatory cytokines. We observed that compared to SumIL2-Fc, ProIL2 149 exhibits markedly reduced serum levels of inflammatory markers IFN $\gamma$ and MCP-1 (Figure 4c150 d). We also used flow cytometry to quantify the expansion of splenic CD8 T cells, which may 151 also contribute to peripheral toxicity. We similarly observed that ProIL2 expands fewer CD8 T 152 cells in the spleen relative to SumIL2-Fc (Figure 4e). Overall, ProIL2 exhibits decreased off- 
153 target immune activation in non-tumor tissues compared to SumIL2-Fc and therefore provides a 154 much wider therapeutic window than IL2-Fc.

155 While ProIL2 reduces immune activation outside of the tumor, we still want to ensure that 156 ProIL2 potently activates intratumor CTLs. We extracted and digested tumors from PBS, 157 SumIL2-Fc, or ProIL2 treated tumor bearing mice 72 hours after treatment. We then used flow 158 cytometry to quantify immune cell levels in the tumor. We observed that both SumIL2-Fc and 159 ProIL2 increased CD8 T cells in the tumor without increasing Tregs, thus inducing a high 160 CD8:Treg ratio (Figure 4f-h). This suggests that ProIL2 induces a strong activating phenotype in 161 the tumor microenvironment (TME). These in vivo experiments demonstrate that ProIL2 162 activates immunity in the TME and minimally stimulates toxic cytokine inflammatory responses 163 in the periphery.

\section{ProIL2 can overcome ICB resistance in advanced cancers}

165 ProIL2 may activate lymphocytes to produce IFN or other cytokines, thus upregulating PD-L1 166 on both host and tumor cells. We aimed to expose whether host or tumor cells respond to IL-2 167 mediated cytokines for PD-L1 expression. We observed that ProIL2 significantly increases the $168 \mathrm{PDL1}^{+} \mathrm{CD}^{+} 5^{+} / \mathrm{CD}^{2} 5^{-}$ratio (Figure 5a, Supplementary Figure 5a-b). We hypothesize that this 169 particular expansion of cell populations would maximize the binding of anti-PDL1 to the PDL1 ${ }^{+}$ 170 host cells that are required for antitumor efficacy of PDL1 blockade ${ }^{22,23}$. We also observe that in $171 \mathrm{CD}^{+} 5^{+}$populations, the MFI level of PDL1 increases more in ProIL2 treated tumors than in PBS

172 treated tumors (Figure 5b, Supplementary Figure 5c). As a result, both the increased frequency

173 and increased expression of PDL1 in host cells in the TME suggest that ProIL2 therapy may 174 sufficiently stimulate immune cells the TME for synergy with anti-PDL1 treatment. 
175 We inoculated mice with B16 tumors and treated these mice with PBS, anti-PDL1, ProIL2, or

176 ProIL2/anti-PDL1. These tumors were resistant to either anti-PDL1 or ProIL2 single therapy, as

177 neither treatment could significantly control these tumors. However, the combination of ProIL2

178 and anti-PDL1 controlled these established tumors much more effectively without causing body

179 weight loss (Figure 5c-d). These data suggest that ProIL2 can effectively overcome resistance to

180 ICB in unresponsive tumors, still without significant toxicity.

Preoperative ProIL2 treatment can eliminate metastatic cancers through an abscopal effect

182 Surgical removal of tumors has remained one of the most effective treatments of primary

183 cancers. However, surgery is not able to cure metastases, and the resulting surgical wound

184 inflammation may potentially even be a trigger for formation of metastatic disease ${ }^{24}$. In order to

185 prevent postoperative metastatic disease, many groups have proposed using neoadjuvant

186 immunotherapy to generate a number of T cells that may be sufficient to patrol and outnumber

187 metastatic tumor cells.

188 We subcutaneously inoculated mice with 4T1 mammary tumors, which spontaneously

189 metastasize to the lung. We preoperatively treated these tumor-bearing mice with either PBS or

190 ProIL2 and resected tumors from half of these mice. Approximately 3 weeks after tumor

191 resection, we extracted lungs from these mice and quantified the number of metastatic nodules

192 and colonies in each lung. We observed that while any single treatment group had many

193 metastatic nodules and colonies, almost no mice in our preoperative ProIL2 and surgery group

194 had any nodules or colonies (Figure 6a-b). We observed that that $80 \%$ of preoperative ProIL2

195 and surgery treated mice survived, whereas the majority of mice in any other treatment group

196 died within the time course of this study (Figure 6c). We also rechallenged these surviving

197 ProIL2/surgery-treated mice with 4T1 tumors at a secondary location and observed that $75 \%$ of 
198 these mice exhibited no tumor relapse. These studies demonstrate that preoperative ProIL2 and

199 surgery treatment is vastly superior to ProIL2 or surgery alone in eradicating metastases and can 200 also prevent cancer recurrence.

201 We subsequently sought to determine how ProIL2 can prevent metastases. One potential model 202 is that ProIL2 can flow into lung tissues and activate residual lymphocytes in the lung to control 203 metastatic disease. Alternatively, TILs activated by ProIL2 are necessary to eradicate metastases. 204 To investigate whether preoperative ProIL2 could generate T cells that migrate into distal sites, 205 we additionally administered FTY720, which binds to sphingosine-1-phosphate receptors in 206 lymph nodes to prevent lymphocyte egress, to preoperative control and ProIL2 treated mice.

207 FTY720 prevents lymphocytes in different tissues (i.e. tumor and lymph nodes) from trafficking 208 into other tissues (i.e. lung). We observed that while combined ProIL2 and surgery treated mice 209 prevented metastatic nodule and colony growth, addition of FTY720 to combined ProIL2 and 210 surgery treatment abrogated this effect (Figure 6a-b). As FTY720 prevents lymphocytes from 211 migrating from treated tissues into the lung, these data suggest that residential lymphocytes in 212 the lung are insufficient for metastatic control, and thus require TILs. These results suggest that 213 neoadjuvant ProIL2 induces an abscopal effect that is effective and required for eradicating 214 metastases.

\section{Discussion}

216 IL-2 was initially considered to be a promising potent antitumor therapy. However, its low half217 life, low tolerability, and induction of cytokine release syndrome significantly limits its use in 218 most human patients. We engineered and generated a unique IL-2 prodrug that combines a 219 potent CD8 T cell preferential IL-2 mutein with a blocking IL-2 receptor that can be released by 220 tumor specific matrix metalloproteinases. This next-generation pro-cytokine incorporates dual 
221 advantages of having potent immunostimulatory activity in the tumor and side effect

222 minimization through tumor specific targeting. Furthermore, our ProIL2 can effectively

223 synergize with current standard of care cancer therapies to overcome ICB resistant and even

224 metastatic cancers.

225 We proposed the concept of an IL-2 prodrug in order to address limitations of not only

226 recombinant IL-2, but also of current next-generation IL-2 designs. One popular IL-2 variant

227 (PEG-IL2) involves attaching PEG to regions of IL-2 that normally bind to IL-2 receptors, thus

228 decreasing peripheral activity of IL-2 ${ }^{9}$. However, production of consistently PEGylated regions

229 of IL-2 is not reliable, and PEG may nonspecifically detach from IL-2. In contrast to PEG-IL2,

230 for which PEGylation and additional conjugation processes may produce mixed products, ProIL2

231 can be produced at a high yield as a single molecule very quickly and efficiently. Therefore,

232 synthesis of ProIL2 would comparatively ease any concerns manufacturers may have about the

233 production and mixed nature of PEG-IL2. ProIL2 is also significantly preferentially active in the 234 tumor.

235 A second IL-2 design fuses IL-2 to an antibody, such as epidermal growth factor receptor, that 236 targets specific proteins on tumor cells. However, upon binding of antibodies to antigens on the 237 tumor surface, bound proteins are endocytosed, potentially before IL-2 in this case can stimulate 238 lymphocytes ${ }^{25}$. Also, even when paired with antibodies, such high affinity IL-2 variants will still 239 target and activate T cells in the periphery. On the other hand, ProIL2 targets MMPs that are 240 secreted by tumor cells or are attached to inflammatory cells in the TME, thus decreasing the 241 concern of endocytosis prior to IL-2 activation of CTLs. Furthermore, antibody/IL-2 fusions 242 require tumors to specifically express the target antigen. Because so many tumors express 
243 MMPs, ProIL2 is not as limited in terms of the expression of a targeted tumor antigen. It is

244 possible that antibody/ProIL2 fusions may further increase tumor targeting.

245 A protease activated IL-2 fusion protein was designed to attempt to block wild-type IL-2 (WT-

246 IL2) with IL2R $\alpha^{26}$. However, this fusion does not contain any modality to extend half-life, such

247 as Fc. Furthermore, this fusion uses WT-IL2 and thus has higher affinity for Tregs when cleaved.

248 To reduce the preferential targeting of IL-2 to Tregs, we designed IL-2 variants with mutations

249 that lower IL2R $\alpha$ binding affinity. IL2R $\alpha$ failed to block this mutant IL-2, likely activating

250 CTLs before reaching tumor tissues. Moreover, a design consisting solely of WT-IL2 and IL2R $\alpha$

251 may dimerize with other WT-IL2/IL2R $\alpha$ complexes, thus potentially decreasing access to the

252 protease substrate linker due to protein structural hindrance ${ }^{27}$. ProIL2 with Fc has a long half-

253 life, high affinity for CD8 T cells, and consists of domains that prevent any evident dimerization.

254 Overall, while many other next-generation IL-2 designs have been tested, they encounter

255 limitations that we address with ProIL2.

256 MMP expression has been also observed to increase as tumors become more invasive or

257 metastatic, and is expressed in a wide range of cancers ${ }^{14,28-31}$. Therefore, we chose to use MMPs

258 based on its specificity and abundance in cancers. Our lab then used mutational screening to

259 synthesize a 10 amino acid peptide substrate that would most effectively cleave in the presence

260 of murine and human tumor fragments. As observed, significant levels of ProIL2 are cleaved in

261 response to MMPs within 1 hour in vitro and inside tumors within 24 hours in vivo (Figure $2 \mathrm{~d}$,

262 3d). This suggests that ProIL2 can favorably become an active cytokine in the tumor quite

263 shortly after injection. Strikingly, very little cleaved ProIL2 was in the liver. The liver can

264 express many proteases such as cathepsins, plasminogen activators, and metalloproteases ${ }^{32-34}$.

265 The liver is heavily involved in clearance of different proteins in the host, and proteinases may 
266 potentially ultimately end up in the liver. The lack of cleaved ProIL 2 in the liver suggests that

267 our MMP substrate is minimally affected by these hepatic proteases and is thus more specific to

268 MMP cleavage.

269 Despite the "release of brakes" by ICB, only a small fraction of patients responds to ICB. For 270 anti-PDL1 in particular, we propose that these patients exhibit resistance to anti-PDL1 for two 271 major reasons. First, some patients may have lower expression of PDL1 in the TME ${ }^{35,36}$. Second, 272 even after anti-PDL1 treatment, limited T cell activating and expanding cytokines in the TME 273 may prevent effector lymphocytes from being fully stimulated. Therefore, T cell growth and 274 activating cytokines are required for rapid and effective T cell activation and expansion, 275 especially after anti-PDL1 treatment. We have observed that ProIL2 can "fuel" immune cells and 276 overcome their dysfunctional state. In particular, anti-PDL1 treatment is dependent on host cell 277 PDL1 expression ${ }^{37,38}$, and ProIL2 is able to both expand the number of host PDL1 ${ }^{+}$cells and 278 increase the expression level of PDL1 on host cells (Figure 5a-b). Furthermore, after anti-PDL1 279 treatment, ProIL2 also provides the follow-up stimulation to effector CD8 T cells in the TME to 280 induce tumor regression (Figure 4f, 5c). Therefore, ProIL2 overcomes anti-PDL1 resistant 281 tumors, thus providing a safe option for synergistic, powerful combination therapy against 282 tumors that may be difficult to treat.

283 Tumor resection is an effective modality for curing patients of primary tumors. However, most 284 of these patients still relapse from metastasis. Immunotherapy can potently prime TILs to egress 285 from tumors and combat metastatic cancer cells, but can often fail to significantly overcome 286 large tumor burdens, especially those that continue to metastasize and disseminate into 287 peripheral tissue ${ }^{39}$. Therefore, the hypothesis that combining neoadjuvant immunotherapy, which 288 would activate and egress a sufficient number of tumor-specific T cells, with surgery can 
289 eradicate a relatively small number of metastatic tumors at distal sites has been proposed.

290 Neoadjuvant immunotherapy, including preoperative IL-2, has improved patient responses to

291 surgery, likely due to improved tumor-specific CTL expansion ${ }^{40-42}$. We also proposed that

292 ProIL2, as a potent tumor targeting immunotherapy, would effectively activate TILs which

293 would be essential for control of metastatic disease. We initially demonstrate that neoadjuvant

294 ProIL2 can indeed eliminate metastatic cancer very effectively (Figure 6). However, when we

295 also inhibited intratumor CTLs from leaving the tumor with FTY720, we observed that ProIL2

296 could no longer control distal lung metastases (Figure 6a-b). These results are consistent with our

297 hypothesis that neoadjuvant immunotherapy can prime and activate tumor specific CTLs which

298 can exit the tumor to eradicate distant metastases.

299 Taken together, our work has designed and synthesized a novel immunocytokine therapy that is

300 safe but still exhibits potent antitumor efficacy. In particular, ProIL2 preferentially targets

301 intratumor CTLs tumor without substantially inducing Tregs or peripheral lymphocytes, thus

302 overcoming a large hurdle for IL-2 therapy. Administration of ProIL2 also overcomes advanced

303 and metastatic tumor resistance to current standard of care therapies. Overall, our study has

304 provided a low toxicity but effective immunotherapy that can overcome clinically relevant

305 advanced cancers and metastasis. 
308 We thank the Institutional Animal Care and Use Committee Animal Resources Center, and Animal 309 Research Center for assistance with welfare and caretaking of all animals. This work was 310 supported by Cancer Prevention and Research Institute of Texas (CPRIT) grant RR150072 given 311 to Yang-Xin Fu, the T32 Integrative Immunology Training Grant, and the T32 Medical Scientist 312 Training Program Training Grant. We also thank Jian Qiao, Zhenhua Ren, Longchao Liu, and 313 Chunbo Dong for providing experiment materials and helpful discussions.

314 Author contributions

315 Conceptualization, E.H., X.C., and Y.-X.F.; Methodology, E.H. and Y.-X.F.; Investigation, E.H., 316 X.C., B.M., J.B. and Z.L.; Writing - Original Draft, E.H.; Writing-Review \& Editing, E.H. and Y.317 X.F.; Funding Acquisition, Y.-X.F.; Resources, X.C. and Z.S.; Supervision, Y.-X.F. 
Figure 1. Engineering ProIL2 Design. a Pipeline for designing and screening the most optimal ProIL2 designs. ProIL2 designs were screened using HEK-Blue ${ }^{\mathrm{TM}}$ IL-2 reporter cell assay, MMP

322 cleavage assay for 4 hours, and tumor inoculation and growth measurement as described in the 323 methods. b Multiple designs of ProIL2 that passed the in vitro screening stage were tested. The Fc 324 arms, listed from $\mathrm{N}$ terminus to $\mathrm{C}$ terminus in each entry, of each proposed design are shown. HEKBlue $^{\mathrm{TM}}$ IL-2 Reporter cell assay was used to determine the $\mathrm{IC}_{50}$ in $\mathrm{nM}$ of functional activity 326 of each ProIL2 design. Fold block of IL2 activity was measured by dividing the IC 50 of each ProIL2 327 design to that of SumIL2-Fc. Each ProIL2 design was cleaved by MMPs and run on SDS-PAGE, 328 where the amount of cleaved vs uncleaved ProIL2 product was quantified. MC38 s.c. tumor 329 bearing mice were injected i.p. with PBS and an equimolar dose of the listed ProIL2 design 9 days after tumor inoculation. 12 days after treatment, tumor volumes of PBS treated and ProIL2 treated mice were measured, and the decrease in tumor volume in ProIL2 treated mice on this day was quantified. Abbreviations: IL2R $\beta=$ IL-2 Receptor Beta, IL2R $\beta *=$ Truncated version of IL-2 Receptor Beta, MMP = 10-mer cleavable MMP substrate, IL2R $\alpha=$ IL2 Receptor Alpha, WTIL2 $334=$ Wild-type IL-2. Data represent the mean \pm s.e.m of replicates.

Figure 2. ProIL2 effectively masks IL-2 activity and is cleaved by MMPs in vitro. a Schematic of ProIL2 depicting that IL2RB binds and blocks IL-2 activity prior to cleavage (left) and activates 337 into SumIL2-Fc upon MMP cleavage (right). b Binding of SumIL2-Fc and ProIL2 at different 338 doses to HEK-Blue ${ }^{\mathrm{TM}}$ IL-2 Reporter cells was assessed via flow cytometry ( $\mathrm{n}=3$ experiments, 3 339 replicates each). c Functional activity of SumIL2-Fc and ProIL2 was assessed by using HEK340 Blue $^{\text {TM }}$ IL-2 Reporter cell assay ( $n=3$ experiments, 3 replicates each). d Protease activation of 341 ProIL2. ProIL2 was incubated with human MMP2, MMP9, and MMP14 for 1 or 4 hours, then run 
on non-reducing SDS-PAGE, where gel band intensity was subsequently quantified ( $\mathrm{n}=3$ experiments). e Functional activity of SumIL2-Fc, ProIL2, and ProIL2 that was incubated with human MMP2, MMP9, and MMP14 for 4 hours was assessed by using HEK-Blue ${ }^{\mathrm{TM}}$ IL-2 Reporter cell assay ( $\mathrm{n}=1$ of 2 experiments, 3 replicates each). Data represent the mean \pm s.e.m of replicates.

\section{Figure 3. ProIL2 preferentially localizes to and cleaves effectively in tumors. a Equimolar} doses of SumIL2-Fc $(20 \mu \mathrm{g})$ or ProIL2 $(30 \mu \mathrm{g})$ was injected i.p. into CT26 s.c. tumor bearing mice, and serum was collected and isolated at multiple time points. hIgG ELISA was used to quantify the amount of SumIL2-Fc or ProIL2 in the serum ( $\mathrm{n}=2$ experiments, 4 replicates each). b-e $20 \mu \mathrm{g}$ ProIL2 was injected i.p. into CT26 s.c. tumor bearing mice, and the labeled tissues were collected and homogenized 24 hours after treatment ( $\mathrm{n}=2$ experiments, 4 replicates each). b hIgG ELISA was used to quantify the amount ProIL2 in each homogenate, normalized by total tissue weight. The quantity within each mouse were then normalized to the amount of ProIL2 in that mouse's tumor. c Cytometric Bead Array was used to quantify the amount of IFN $\gamma$ in each tissue homogenate. d-e hIgG immunoprecipitation of equivalent weights of tissue homogenate was performed with Protein A binding beads, and then run on Western Blot with hIgG binding antibody. d Relative gel band intensity per lane was quantified. e Western Blot relative gel band intensities were quantified, and within each mouse, the total percentage of cleaved ProIL2 between each tissue was compared and quantified. Data represent the mean \pm s.e.m of replicates.

Figure 4. In vivo assessment of ProIL2. a-b MC38 s.c. tumor bearing mice were injected i.p. with one dose of the labeled treatment on day 9 post tumor inoculation; tumor growth and body weight were measured ( $\mathrm{n}=2$ experiments, 3-4 replicates each). c-e B16 s.c. tumor bearing mice were injected i.p. with PBS, SumIL2-Fc or ProIL2 (120 pmol) one time on day 9 post tumor inoculation ( $\mathrm{n}=2$ experiments, 3-4 replicates each). Serum was collected and isolated from mice 
24 hours post treatment, and Cytometric Bead Array was used to quantify the amount of serum IFN $\gamma$ or MCP-1. e 72 hours after treatment, mice were euthanized and spleens were extracted and resuspended as single cells. Flow cytometry was used to quantify the amount of CD8 T cells in each respective group. f-h B16 s.c. tumor bearing mice were injected i.p. with PBS, SumIL2-Fc or ProIL2 (300 pmol) one time on day 9 post tumor inoculation $(n=4$ experiments, 4 replicates each). 72 hours after treatment, mice were euthanized and tumors were extracted, digested in collagenase/DNAse, and resuspended as single cells. Flow cytometry was used to quantify the amount of CD8 T cells, amount of Tregs, and CD8:Treg ratio in each respective group. Data represent the mean \pm s.e.m. of replicates.

Figure 5. ProIL2 can overcome advanced tumor resistance to ICB. a-b B16 s.c. tumor bearing mice were injected i.p. with PBS or ProIL2 (300 pmol) one time on day 9 post tumor inoculation. 72 hours after treatment, mice were euthanized and tumors were extracted, digested in collagenase/DNAse, and resuspended as single cells. Flow cytometry was used to quantify the amount of $\mathrm{PDL}^{+}$and $\mathrm{CD}^{+} 5^{+}$cells. In $\mathrm{CD}^{4} 5^{+}$cells, the MFI of $\mathrm{PDL1}^{+}$and $\mathrm{PDL1}^{-}$cells were compared ( $\mathrm{n}=3$ experiments, 4 replicates each). c-d B16 s.c. tumor bearing mice were injected i.p. with PBS, anti-PDL1 (200 $\mu$ g each), ProIL2 (300 pmol), or anti-PDL1 and ProIL2 one time on day 9 post tumor inoculation; tumor growth and body weight were measured $(n=1$ of 2 experiments, 3-4 replicates each). Data represent the mean \pm s.e.m. of replicates.

\section{Figure 6. Preoperative ProIL2 combined with surgery can eliminate metastases through an} abscopal effect. 4T1 s.c. tumor bearing mice were injected i.p. with PBS or ProIL2 (300 pmol) on day 9 and 12 post tumor inoculation. Mice treated with FTY720 were i.p. treated with $10 \mu \mathrm{g}$ every other day for 14 days starting on day 8 post tumor inoculation. On day 14 post tumor inoculation, the listed mice had their tumors surgically removed. a 25 days post initial treatment, mice were 
388 euthanized and metastatic nodules in the thoracic cavity were counted. b 25 days post initial 389 treatment, mice were euthanized and lungs were extracted, digested in collagenase/DNAse, and 390 resuspended as single cells. Resuspended cells were plated at a 1:400 ratio with 6-thioguanine 391 selection medium, and the number of colonies were counted 6 days post seeding ( $\mathrm{n}=2$ experiments, 392 2-5 replicates each). c Mice were analyzed for survival starting the day after the first i.p. injection 393 treatment ( $\mathrm{n}=2$ experiments, 5 replicates each). Data represent the mean \pm s.e.m. of replicates. 
Mice

397 Female C57BL/6J and BALB/c mice were purchased from The Jackson Laboratory. All mice were 398 maintained under specific pathogen-free conditions. Animal care and experiments were carried out 399 under institutional and National Institutes of Health protocol and guidelines. This study has been 400 approved by the Institutional Animal Care and Use Committee of the University of Texas 401 Southwestern Medical Center.

\section{Cell lines and reagents}

403 B16, MC38, CT26, and 4T1 cell lines were purchased from American Type Culture Collection 404 (ATCC). All cell lines were routinely tested using mycoplasma contamination kit (R\&D) and 405 cultured in Dulbecco's modified Eagle's medium supplemented with $10 \%$ heat-inactivated fetal 406 bovine serum, $100 \mathrm{U} / \mathrm{ml}$ penicillin, and $100 \mathrm{U} / \mathrm{ml}$ streptomycin under $5 \% \mathrm{CO} 2$ at $37{ }^{\circ} \mathrm{C}$. Anti407 PDL1 (Atezolizumab) mAb was purchased from Genentech. FTY720 was purchased from 408 Selleckchem

\section{Flow Cytometry Analysis}

410 Single cell suspensions from either spleen, tumor or in vitro co-cultured cells were incubated with 411 anti-Fc $\gamma \mathrm{III} / \mathrm{II}$ receptor (clone $2.4 \mathrm{G} 2$ ) for 15 minutes to block non-specific binding before staining

412 with the conjugated antibodies. Fixable Viability Dye eFluor ${ }^{\mathrm{TM}} 506$ was used to exclude dead cells. 413 Foxp3 was stained intracellularly by using True-Nuclear transcription factor buffer set (BioLegend) 414 following the manufacturer's instructions. 
415 To assess ProIL2 and SumIL2-Fc binding affinity, HEK-Blue ${ }^{\mathrm{TM}}$ IL-2 cells (InvivoGen) were first 416 stained with various dilutions of ProIL2 or SumIL2-Fc, then PE conjugated donkey anti-human

417 IgG was used as a secondary antibody. All staining steps were conducted at $4{ }^{\circ} \mathrm{C}$ in the dark. $\mathrm{BD}^{\mathrm{TM}}$ 418 Cytometric Bead Array (CBA) Mouse Inflammation Kit was used to measure the cytokines in the 419 supernatants from mouse serum or tissue according to the manufacturer's protocol (BD 420 Biosciences). Data were collected on CytoFLEX flow cytometer (Beckman Coulter, Inc) and 421 analyzed by using CytExpert (Beckman Coulter, Inc) or FlowJo (Tree Star Inc., Ashland, OR) 422 software.

\section{Enzyme-Linked ImmunoSorbent Assay (ELISA)}

424 Microtiter plates (Corning Costar) were coated with $2 \mu \mathrm{g} / \mathrm{mL}(100 \mu \mathrm{L} /$ well) capture antibody 425 (AffiniPure Goat Anti-Human IgG, Fc $\gamma$ fragment specific) overnight at $4{ }^{\circ} \mathrm{C}$. After washing and 426 blocking, diluted tissue lysate or serum from treated mice were added and incubated at $37^{\circ} \mathrm{C}$ for $4271 \mathrm{hr}$. After washing, Alkaline Phosphatase (AP) conjugated Goat Anti-Human IgG (Fc $\gamma$ fragment 428 specific) was added and incubated at $37^{\circ} \mathrm{C}$ for 30 minutes. Finally, the plates were visualized by 429 adding $100 \mu \mathrm{L}$ KPL Diethanolamine + pNPP substrate (Seracare Life Sciences) and read at 405 430 nm using the SPECTROstar Nano (BMG LABTECH).

\section{Tumor growth and treatment}

432 A total of $1 \times 10^{6} \mathrm{MC} 38,3 \times 10^{5} \mathrm{~B} 16,5 \times 10^{5} \mathrm{CT} 26$, or $5 \times 10^{5}$ 4T1 cells were inoculated 433 subcutaneously into right dorsal flanks of the mice in $100 \mu \mathrm{L}$ phosphate buffered saline (PBS).

434 Tumor-bearing mice were randomly grouped into treatment groups when tumors grew to around $43570-100 \mathrm{~mm}^{3}$. For SumIL2-Fc treatment in MC38/B16/CT26 tumor bearing mice, one dose of 1 $436 \mathrm{mg} / \mathrm{kg}(300 \mathrm{pmol}$ or $20 \mu \mathrm{g})$ was intraperitoneally given on day 9. For ProIL2 treatment in 
437 MC38/B16/CT26 tumor bearing mice, one dose of $1.5 \mathrm{mg} / \mathrm{kg}(300 \mathrm{pmol}$ or $30 \mu \mathrm{g})$ was 438 intraperitoneally given on day 9. For ProIL2 treatment in 4T1 tumor bearing mice, two doses of $4391.5 \mathrm{mg} / \mathrm{kg}(300 \mathrm{pmol}$ or $30 \mu \mathrm{g})$ was intraperitoneally given on days 9 and 12 . For surgical treatment, 440 tumors and surrounding skin were removed on day 14. For FTY720 treatment, $10 \mu \mathrm{g}$ FTY720 was 441 intraperitoneally administrated one day before ProIL2 treatment initiation and then $10 \mu \mathrm{g}$ every 442 other day for 2 weeks or until experiment termination. $200 \mu \mathrm{g}$ anti-PDL1 was administered 443 concurrently with ProIL2 treatment. For rechallenge experiments, $2.5 \times 10^{6} 4 \mathrm{~T} 1$ cells were 444 inoculated subcutaneously into left dorsal flanks of mice in $100 \mu \mathrm{L}$ PBS. Tumor volumes were 445 measured by the length (a), width (b) and height (h) and calculated as tumor volume $=a b h / 2$.

446 Production of Fusion Proteins

447 Based on the heterodimeric Fc variant KiHss-AkKh platform as previously described, sumIL2 was 448 fused with knob variant Fc region, and the IL2R $\beta$ /MMP linker was fused with hole variant Fc 449 region. ProIL2 and SumIL2-Fc were generated by transient co-transfection of two arms of 450 plasmids into FreeStyle ${ }^{\mathrm{TM}} 293-\mathrm{F}$ cells. The supernatant containing fusion proteins was purified 451 using Protein A affinity chromatography according to the manufacturer's protocol. The 452 heterogeneity and purity were confirmed by SDS-PAGE.

\section{Tissue Homogenate Preparation}

454 Spleen, lung, liver and tumor were excised one day after ProIL2 treatment and homogenized in 455 the Cell Lysis Kit (Bio-Rad Laboratories) with the FastPrep-24 5G Homogenizer. The samples 456 were centrifuged for 10 minutes at $13000 \mathrm{rpm}$, and the supernatant was collected.

\section{Tumor Digestion}


458 Tumor tissues were excised and digested with $1 \mathrm{mg} / \mathrm{mL}$ Collagenase I (Sigma) and $0.5 \mathrm{mg} / \mathrm{mL}$

459 DNase I (Roche) in the $37^{\circ} \mathrm{C}$ for $30 \mathrm{mins}$, tumor was then passed through a $70 \mu \mathrm{m}$ cell strainer to

460 remove large pieces of undigested tumor. Tumor infiltrating cells were washed twice with PBS

461 containing $2 \mathrm{mM}$ EDTA.

\section{IL-2 Reporter Cell Culture Assay}

463 Various dilutions of IL-2 fusion protein variants were incubated with $5 \times 10^{4}$ HEK-Blue $^{\mathrm{TM}}$ IL-2

464 cells (InvivoGen) for 24 hours as per manufacturer's protocol. Afterwards, $25 \mu \mathrm{L}$ cell supernatant 465 was collected and incubated with $75 \mu \mathrm{L}$ Quanti-Blue reagent (InvivoGen) for 1 hour. The mixture 466 was read at $650 \mathrm{~nm}$ using the SPECTROstar Nano (BMG LABTECH).

\section{MMP cleavage of ProIL2 and SDS-PAGE}

468 Recombinant human MMP2 and MMP9 (BioLegend) were activated with 1mM 4-

469 Aminophenylmercuric acetate for 1 hour as per manufacturer's instructions. Recombinant human 470 MMP14 (R\&D Sciences) was activated with $0.1 \mu \mathrm{g} / \mathrm{mL}$ rhTrypsin (R\&D Sciences) for 1 hour 471 and then incubated with $1 \mathrm{ng} / \mu \mathrm{L}$ AEBSF (R\&D Sciences) for 15 minutes as per manufacturer's 472 instructions. Cleavage Assay Buffer consisted of $50 \mathrm{mM}$ Tris, $10 \mathrm{mM} \mathrm{CaCl}_{2}, 150 \mathrm{mM} \mathrm{NaCl}$, and $473 \quad 0.05 \%(w / v)$ Brij 35 at $\mathrm{pH}$ 7.5. $50 \mathrm{ng}$ MMP2, $50 \mathrm{ng}$ MMP9, and $100 \mathrm{ng}$ MMP14 were incubated 474 with $1.5 \mu \mathrm{g}$ ProIL2 in Cleavage Assay Buffer at $37^{\circ} \mathrm{C}$ for 1 or 4 hours. Proteins were then run on 475 SDS-PAGE and relative gel band intensities were analyzed to quantify the amount of cleavage in 476 each ProIL2/MMP mixture.

477 Tissue Homogenate Immunoprecipitation and Western Blot Assay

478 Tumor, liver, lung, and spleen tissues were homogenized as described above. Equivalent 479 amounts of tissue homogenate by weight were incubated with Protein A binding beads at a 100:1 
480 ratio for 12 hours at $4^{\circ} \mathrm{C}$. The beads were then isolated and run on a non-reducing SDS-PAGE.

481 Proteins were transferred onto membrane using Trans-Blot Turbo manufacturer instructions. The 482 membrane was incubated and rotated on shaker with 5\% milk in Phosphate Buffered Saline with $483 \quad 0.1 \%$ Tween 20 Detergent for 1 hour. After washing, Horseradish Peroxidase (HRP) conjugated 484 Goat Anti-Human IgG $(\mathrm{H}+\mathrm{L})$ was added at a 1:2000 dilution and rotated on shaker for 1 hour. 485 The membrane was developed using SuperSignal West Dura Extended Duration Substrate and 486 imaged with ChemiDoc Touch Imaging System (BioRad).

\section{4T1 Metastatic Tumor Colonogenic Assay}

488 Lung tissues from 4T1 bearing or tumor resected mice were excised and digested with $1 \mathrm{mg} / \mathrm{mL}$ 489 Collagenase I (Sigma) and $0.5 \mathrm{mg} / \mathrm{mL}$ DNase I (Roche) at $37^{\circ} \mathrm{C}$ for $30 \mathrm{mins}$, and then passed 490 through a $70 \mu \mathrm{m}$ cell strainer to remove large pieces of undigested tumor. Lungs cells were 491 resuspended in $3 \mathrm{~mL}$ Dulbecco's modified Eagle's medium supplemented with 10\% heat492 inactivated fetal bovine serum, $100 \mathrm{U} / \mathrm{ml}$ penicillin, $100 \mathrm{U} / \mathrm{ml}$ streptomycin, and $10 \mu \mathrm{g} / \mathrm{mL} 6-$ 493 thioguanine. The cells were plated in 6 well plates at a 1:400 dilution. After 6 days, cells were 494 stained with crystal violet staining solution and colonies were counted.

\section{$495 \quad$ Statistical analysis}

496 All the data analyses were performed with GraphPad Prism statistical software and shown as mean $497 \pm$ SEM. P value was determined by two-way ANOVA for tumor growth, Log-rank test for survival, 498 or unpaired two-tailed t-tests for other analysis. A value of $\mathrm{p}<0.05$ was considered statistically 499 significant. For notation on figures, $*$ corresponds with $\mathrm{p}<0.05, * *$ with $\mathrm{p}<0.005$, and $* * *$ with $500 \quad \mathrm{p}<0.0005$.

\section{KEY RESOURCES TABLE}




\begin{tabular}{|c|c|c|}
\hline REAGENT or RESOURCE & SOURCE & IDENTIFIER \\
\hline \multicolumn{3}{|l|}{ Antibodies } \\
\hline Anti-PDL1 Atezolizumab & Genentech & N/A \\
\hline Anti-CD45 (Flow cytometry, 30-F11) & BioLegend & Cat\# 103126 \\
\hline Anti-CD8 (Flow cytometry, 53-6.7) & BioLegend & Cat\# 100730 \\
\hline Anti-CD4 (Flow cytometry, RM4-5) & BD Biosciences & Cat\# 550954 \\
\hline Anti-Foxp3 (Flow cytometry, MF-14) & BioLegend & Cat\# 126408 \\
\hline Anti-PD-L1 (Flow cytometry, 10F.9G2) & BioLegend & Cat\# 124308 \\
\hline Fixable Viability Dye eFluor ${ }^{\mathrm{TM}} 506$ & Thermo Fisher & Cat\# 65-0866-18 \\
\hline Anti-FcyIII/II receptor (clone 2.4G2) & BD Biosciences & Cat\# 553141 \\
\hline Goat Anti-Human IgG-HRP & Santa Cruz Biotech & Cat\# sc-2453 \\
\hline $\begin{array}{l}\text { AffiniPure Goat Anti-Human IgG, Fc } \gamma \text { fragment } \\
\text { specific }\end{array}$ & $\begin{array}{l}\text { Jackson } \\
\text { ImmunoResearch }\end{array}$ & Cat\# 109-005-098 \\
\hline $\begin{array}{l}\text { Alkaline Phosphatase-AffiniPure Goat Anti- } \\
\text { Human IgG, Fc } \gamma \text { fragment specific }\end{array}$ & $\begin{array}{l}\text { Jackson } \\
\text { ImmunoResearch }\end{array}$ & Cat\# 109-055-098 \\
\hline Donkey Anti-Human IgG $(\mathrm{H}+\mathrm{L})$ & $\begin{array}{l}\text { Jackson } \\
\text { ImmunoResearch }\end{array}$ & Cat\# 709-116-149 \\
\hline \multicolumn{3}{|l|}{ Bacterial and Virus Strains } \\
\hline \multicolumn{3}{|l|}{$\mathrm{N} / \mathrm{A}$} \\
\hline \multicolumn{3}{|l|}{ Biological Samples } \\
\hline \multicolumn{3}{|l|}{ N/A } \\
\hline \multicolumn{3}{|l|}{ Chemicals, Peptides, and Recombinant Proteins } \\
\hline FTY720 (hydrochloride) & Selleckchem & Cat\# S5002 \\
\hline Dulbecco's Modified Eagle's Medium & Sigma- Aldrich & Cat\# D6429 \\
\hline Collagenase type I & Sigma & Cat\# C0130 \\
\hline DNase I & Roche & Cat\# 11284932001 \\
\hline 6-Thioguanine, 98\%, Alfa Aesar & Thermo Fisher & Cat\# AAB2128003 \\
\hline Recombinant Human MMP-2 (carrier-free) & BioLegend & Cat\# 554302 \\
\hline Recombinant Human MMP-9 (carrier-free) & BioLegend & Cat\# 550502 \\
\hline 4-Aminophenylmercuric acetate & Sigma- Aldrich & Cat\# A9563 \\
\hline $\begin{array}{l}\text { Recombinant Human MMP-14/MT1-MMP } \\
\text { (NS0-expressed) Protein, CF }\end{array}$ & R\&D Systems & $\begin{array}{l}\text { Cat\# 918-MPN- } \\
010\end{array}$ \\
\hline $\begin{array}{l}\text { Recombinant Human Active Trypsin 3/PRSS3 } \\
\text { Protein, CF }\end{array}$ & R\&D Systems & Cat\# 3714-SE-010 \\
\hline AEBSF, CF & R\&D Systems & Cat\# EI001 \\
\hline 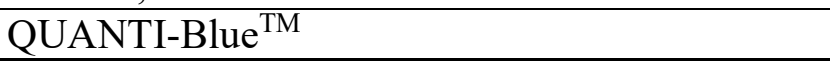 & InvivoGen & Cat\# rep-qbs \\
\hline \multicolumn{3}{|l|}{ Critical Commercial Assays } \\
\hline $\begin{array}{l}\text { BD TM Cytometric Bead Array (CBA) Mouse } \\
\text { Inflammation Kit }\end{array}$ & BD Biosciences & Cat\# 552364 \\
\hline True-Nuclear ${ }^{\mathrm{TM}}$ Transcription Factor Buffer Set & BioLegend & Cat\# 424401 \\
\hline pNPP Microwell Substrate System & Seracare Life Sciences & Cat\# 50-80-00 \\
\hline \multicolumn{3}{|l|}{ Deposited Data } \\
\hline $\mathrm{N} / \mathrm{A}$ & & \\
\hline Experimental Models: Cell L & & \\
\hline
\end{tabular}




\begin{tabular}{|c|c|c|}
\hline B16 & ATCC & Cat\# CRL-6322 \\
\hline MC38 & ATCC & N/A \\
\hline CT26 & ATCC & Cat\# CRL-2638 \\
\hline 4T1 & ATCC & Cat\# CRL-2539 \\
\hline FreeStyle $^{\mathrm{TM}} 293-\mathrm{F}$ & Thermo Fisher & Cat\# R79007 \\
\hline HEK-Blue ${ }^{\mathrm{TM}}$ IL-2 Cells & InvivoGen & Cat\# hkb-il2 \\
\hline \multicolumn{3}{|c|}{ Experimental Models: Organisms/Strains } \\
\hline $\mathrm{C} 57 \mathrm{BL} / 6 \mathrm{~J}$ & Jackson Laboratory & Cat\# 000664 \\
\hline $\mathrm{BALB} / \mathrm{c}$ & Jackson Laboratory & Cat\# 000651 \\
\hline \multicolumn{3}{|l|}{ Oligonucleotides } \\
\hline \multicolumn{3}{|l|}{ N/A } \\
\hline \multicolumn{3}{|l|}{ Recombinant DNA } \\
\hline Plasmid: pEE6.4-IL2R $\beta-M M P-F c 9$ & This paper & N/A \\
\hline Plasmid: pEE6.4-SumIL2-Fc6 & This paper & N/A \\
\hline Plasmid: pEE6.4-Fc9 & This paper & N/A \\
\hline \multicolumn{3}{|l|}{ Software and Algorithms } \\
\hline GraphPad Prism software 7.0 & GraphPad Software, Inc. & $\begin{array}{l}\text { https://graphpad.co } \\
\text { m/scientific- } \\
\text { software/prism/ }\end{array}$ \\
\hline CytExpert & Beckman Coulter, Inc & $\begin{array}{l}\text { https://www.beck } \\
\text { man.com/coulter- } \\
\text { flow- } \\
\text { cytometers/cytofle } \\
\text { x/cytexpert }\end{array}$ \\
\hline FlowJo & Tree Star Inc. & $\begin{array}{l}\text { https://www.flowj } \\
\text { o.com/solutions/flo } \\
\text { wjo }\end{array}$ \\
\hline Image Lab ${ }^{\mathrm{TM}}$ Software & Bio-Rad & $\begin{array}{l}\text { http://www.bio- } \\
\text { rad.com/en- } \\
\text { us/category/ } \\
\text { image-analysis- } \\
\text { software }\end{array}$ \\
\hline \multicolumn{3}{|l|}{ Other } \\
\hline N/A & & \\
\hline
\end{tabular}




\section{References}

504 1. Liao, W., Lin, J.-X. \& Leonard, W. J. Interleukin-2 at the Crossroads of Effector

505 Responses, Tolerance, and Immunotherapy. Immunity 38, 13-25 (2013).

506 2. Rosenberg, S. A. Interleukin 2 for patients with renal cancer. Nat. Clin. Pract. Oncol. 4, $507 \quad$ 497-497 (2007).

508 3. Vignali, D. A. A. Mechanisms of T(reg) Suppression: Still a Long Way to Go. Front. $509 \quad$ Immunol. 3, 191 (2012).

510 4. Donohue, J. H. \& Rosenberg, S. A. The fate of interleukin-2 after in vivo administration. 511 J. Immunol. 130, 2203-8 (1983).

512 5. López-Soto, A., Gonzalez, S., Smyth, M. J. \& Galluzzi, L. Control of Metastasis by NK $513 \quad$ Cells. Cancer Cell 32, 135-154 (2017).

514 6. Reddy, M., Eirikis, E., Davis, C., Davis, H. M. \& Prabhakar, U. Comparative analysis of 515 lymphocyte activation marker expression and cytokine secretion profile in stimulated 516 human peripheral blood mononuclear cell cultures: an in vitro model to monitor cellular 517 immune function. J. Immunol. Methods 293, 127-142 (2004).

518 7. Wang, X., Rickert, M. \& Garcia, K. C. Structure of the quaternary complex of interleukin5192 with its alpha, beta, and gammac receptors. Science 310, 1159-63 (2005).

520 8. Vazquez-Lombardi, R. et al. Potent antitumour activity of interleukin-2-Fc fusion proteins 521 requires Fc-mediated depletion of regulatory T-cells. Nat. Commun. 8, 1-12 (2017).

522 9. Charych, D. H. et al. NKTR-214, an Engineered Cytokine with Biased IL2 Receptor Binding, Increased Tumor Exposure, and Marked Efficacy in Mouse Tumor Models. Clin. 
Cancer Res. 22, 680-690 (2016).

525 10. Levin, A. M. et al. Exploiting a natural conformational switch to engineer an interleukin-2 $526 \quad$ 'superkine'. Nature 484, 529-533 (2012).

527 11. Klein, C. et al. Cergutuzumab amunaleukin (CEA-IL2v), a CEA-targeted IL-2 variant528 based immunocytokine for combination cancer immunotherapy: Overcoming limitations

531 12. Sun, Z. et al. A next-generation tumor-targeting IL-2 preferentially promotes tumor532 infiltrating CD8+ T-cell response and effective tumor control. Nat. Commun. 10, 1-12 (2019).

13. Deryugina, E. I. \& Quigley, J. P. Matrix metalloproteinases and tumor metastasis. Cancer 535 and Metastasis Reviews 25, 9-34 (2006).

14. Gobin, E. et al. A pan-cancer perspective of matrix metalloproteases (MMP) gene expression profile and their diagnostic/prognostic potential. BMC Cancer 19, 581 (2019).

538 15. Wei, H. et al. Structural basis of a novel heterodimeric Fc for bispecific antibody production. Oncotarget 8, 51037-51049 (2017).

540 16. Lo, M. et al. Effector-attenuating substitutions that maintain antibody stability and reduce toxicity in mice. J. Biol. Chem. 292, 3900-3908 (2017).

542 17. Lotze, M. T. et al. In vivo administration of purified human interleukin 2. II. Half life, 543 immunologic effects, and expansion of peripheral lymphoid cells in vivo with recombinant IL 2. J. Immunol. 135, 2865-75 (1985). 
545 18. Anderson, P. M. \& Sorenson, M. A. Effects of Route and Formulation on Clinical

546 Pharmacokinetics of Interleukin-2. Clinical Pharmacokinetics 27, 19-31 (1994).

547 19. Bruserud, Ø. et al. IFN- $\gamma$ and TNF- $\alpha$ secretion by CD4+ and CD8+ TCR $\alpha \beta+$ T-cell clones 548 derived early after allogeneic bone marrow transplantation. Eur. J. Haematol. 51, 73-79

$549 \quad$ (1993).

550 20. Brehm, M. A., Daniels, K. A. \& Welsh, R. M. Rapid Production of TNF- $\alpha$ following TCR 551 Engagement of Naive CD8 T Cells. J. Immunol. 175, 5043-5049 (2005).

552 21. Bhat, P., Leggatt, G., Waterhouse, N. \& Frazer, I. H. Interferon- $\gamma$ derived from cytotoxic 553 lymphocytes directly enhances their motility and cytotoxicity. Cell Death Dis. 8, e2836 $554 \quad(2017)$

555 22. Tang, H. et al. PD-L1 on host cells is essential for PD-L1 blockade-mediated tumor 556 regression. J. Clin. Invest. 128, 580-588 (2018).

557 23. Juneja, V. R. et al. PD-L1 on tumor cells is sufficient for immune evasion in immunogenic tumors and inhibits CD8 T cell cytotoxicity. J. Exp. Med. 214, 895-904 (2017).

560 24. Tohme, S., Simmons, R. L. \& Tsung, A. Surgery for cancer: A trigger for metastases. 561 Cancer Research 77, 1548-1552 (2017).

562 25. Mellman, I. \& Yarden, Y. Endocytosis and cancer. Cold Spring Harb. Perspect. Biol. 5, 563 (2013).

564 26. Puskas, J. et al. Development of an attenuated interleukin-2 fusion protein that can be 565 activated by tumour-expressed proteases. Immunology 133, 206-220 (2011). 
566 27. Ward, N. C. et al. IL-2/CD25: A Long-Acting Fusion Protein That Promotes Immune

567 Tolerance by Selectively Targeting the IL-2 Receptor on Regulatory T Cells. J. Immunol.

$568 \quad 201,2579-2592(2018)$.

569 28. Sullu, Y., Demirag, G. G., Yildirim, A., Karagoz, F. \& Kandemir, B. Matrix

570 metalloproteinase-2 (MMP-2) and MMP-9 expression in invasive ductal carcinoma of the

$571 \quad$ breast. Pathol. - Res. Pract. 207, 747-753 (2011).

572 29. Gurgel, D. C. et al. Immunoexpression of Metalloproteinases 2 and 14 and TIMP-2

573 Inhibitor in Main Types of Primary Gastric Carcinomas and Lymph Node Metastasis.

$574 \quad$ Pathol. Oncol. Res. 21, 73-81 (2015).

575 30. Lu, L. S., Chen, L., Ding, W. X., Li, K. \& Wu, J. J. Elevated expression of both MDR1 576 and MMP-2 genes in metastasized lymph node of invasive ductal breast cancer. Eur. Rev.

577 Med. Pharmacol. Sci. 16, 2037-2043 (2012).

578 31. García, M. F. et al. Comparative study of the expression of metalloproteases and their 579 inhibitors in different localizations within primary tumours and in metastatic lymph nodes 580 of breast cancer. Int. J. Exp. Pathol. 91, 324-334 (2010).

581 32. Niiya, M. et al. Increased ADAMTS-13 proteolytic activity in rat hepatic stellate cells 582 upon activation in vitro and in vivo. J. Tissue Eng. Regen. Med. 4, 1063-1070 (2006).

583 33. Manchanda, M. et al. Cathepsin L and B as Potential Markers for Liver Fibrosis: Insights 584 From Patients and Experimental Models. Clin. Transl. Gastroenterol. 8, e99 (2017).

585 34. Shanmukhappa, K., Sabla, G. E., Degen, J. L. \& Bezerra, J. A. Urokinase-type 586 plasminogen activator supports liver repair independent of its cellular receptor. $B M C$ 
Gastroenterol. 6, 40 (2006).

588 35. Ren, D. et al. Predictive biomarkers and mechanisms underlying resistance to PD1/PD-L1 $589 \quad$ blockade cancer immunotherapy. Molecular Cancer 19, 19 (2020).

590 36. Cottrell, T. R. \& Taube, J. M. PD-L1 and Emerging Biomarkers in Immune Checkpoint 591 Blockade Therapy. Cancer Journal (United States) 24, 41-46 (2018).

592 37. Peng, Q. et al. PD-L1 on dendritic cells attenuates T cell activation and regulates response 593 to immune checkpoint blockade. Nat. Commun. 11, 1-8 (2020).

594 38. Lin, H. et al. Host expression of PD-L1 determines efficacy of PD-L1 pathway blockade595 mediated tumor regression. J. Clin. Invest. 128, 805-815 (2018).

39. Turcotte, S. \& Rosenberg, S. A. Immunotherapy for Metastatic Solid Cancers. Advances in Surgery 45, 341-360 (2011).

40. Romano, F. et al. Biological, histological, and clinical impact of preoperative IL-2 administration in radically operable gastric cancer patients. J. Surg. Oncol. 88, 240-247

600 (2004).

601 41. Angelini, C. et al. Preoperatiye interleukin-2 immunotherapy in pancreatic cancer: Preliminary results. Hepatogastroenterology. 53, 141-144 (2006).

42. Liu, J. et al. Improved efficacy of neoadjuvant compared to adjuvant immunotherapy to 604 eradicate metastatic disease. Cancer Discov. 6, 1382-1399 (2016).

605 
a

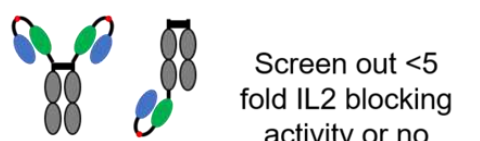
MMP cleavage<smiles>CC1CC2CCCC(C2)O1</smiles><smiles>CC1CC2CCC(CC2C)O1</smiles>

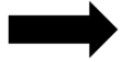

80

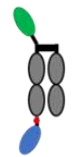

In Vitro Screening

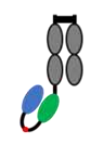

8

80
Screen for highest antitumo efficacy

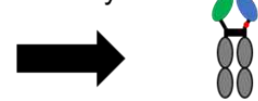

In Vivo Screening

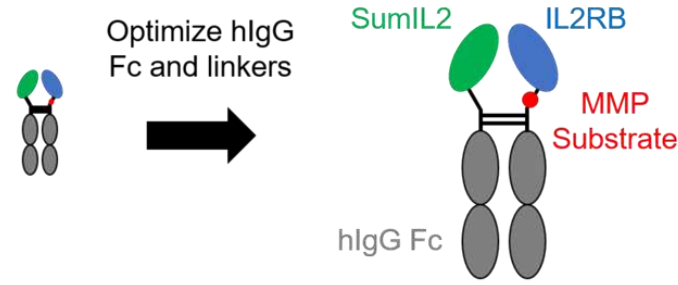

Final ProlL2

b

\begin{tabular}{|c|c|c|c|c|c|}
\hline Arm 1 & Arm 2 & IC50 & Fold Block of IL2 Activity & $\%$ Cleavage & $\%$ Tumor Reduction \\
\hline SumlL2-Fc & Fc & $43.9 \pm 4.5$ & N/A & N/A & $93.8 \pm 2.8$ \\
\hline IL2R $\beta-F c$ & SumlL2-Fc & $1072.6 \pm 115.8$ & 24.4 & 0 & $4.5 \pm 17.0$ \\
\hline IL2Rß-MMP-Fc & SumIL2-Fc & $371.6 \pm 50.0$ & 8.5 & 66.4 & $89.8 \pm 3.0$ \\
\hline IL2Rß-MMP-IL2R $\alpha-F c$ & WTIL2-Fc & $360.7 \pm 24.2$ & 8.2 & 52.8 & $75.5 \pm 11.5$ \\
\hline IL2R $\beta^{*}-M M P-S u m I L 2-F c$ & Fc & $347.4 \pm 17.9$ & 7.9 & 29.1 & $56.6 \pm 10.2$ \\
\hline IL2Rß-MMP-SumIL2-Fc & Fc & $846.1 \pm 40.0$ & 19.3 & 14 & $54.3 \pm 11.7$ \\
\hline Fc-SumIL2-MMP-IL2Rß & Fc & $1301.0 \pm 229.5$ & 29.5 & 15.8 & $24.5 \pm 19.8$ \\
\hline IL2Rß-MMP-IL2R $\alpha-W T I L 2-F c$ & Fc & $4837.7 \pm 1032.6$ & 110.0 & 28.6 & $21.8 \pm 25.1$ \\
\hline
\end{tabular}


Figure 2

a

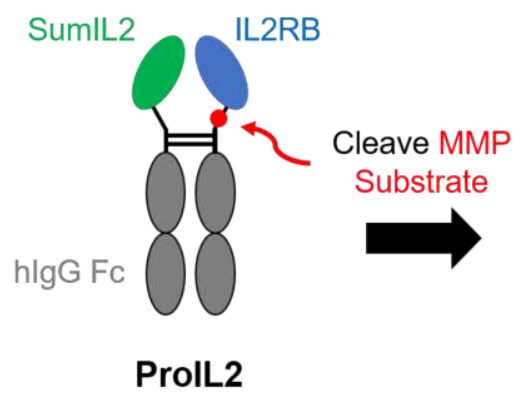

b

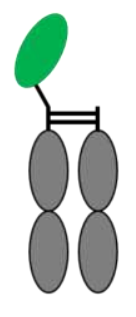

SumIL2-Fc

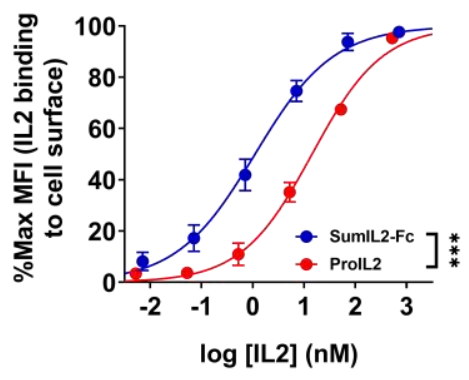

c

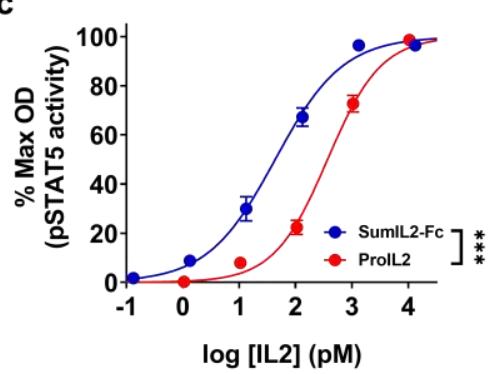

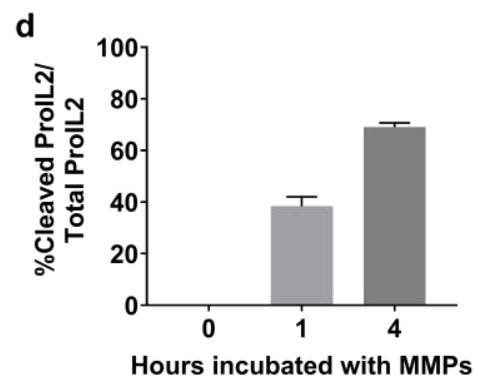

e

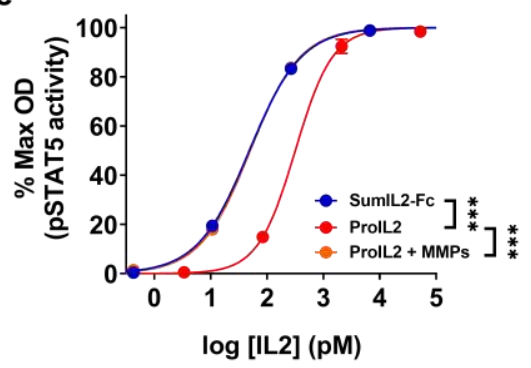

611 
Figure 3

a

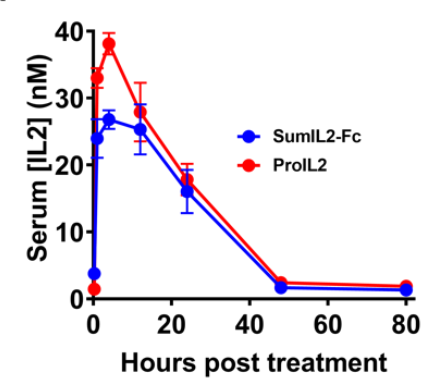

b

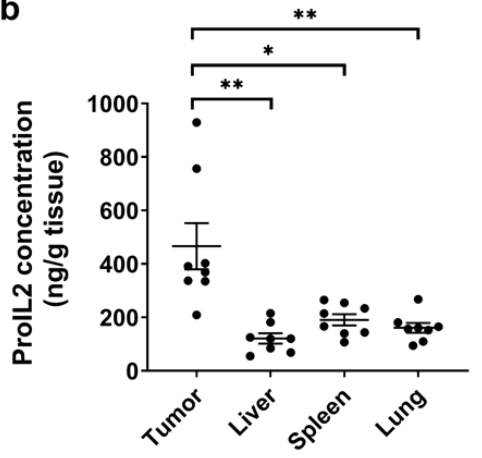

c

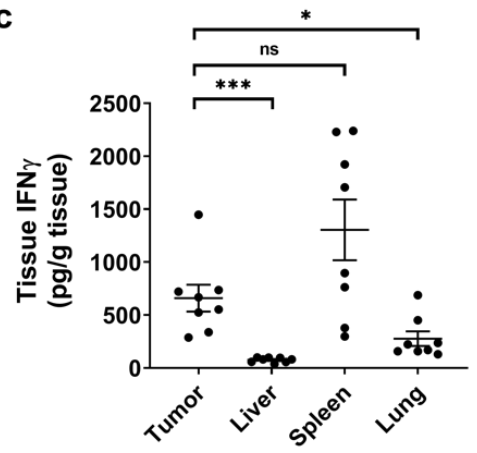

d

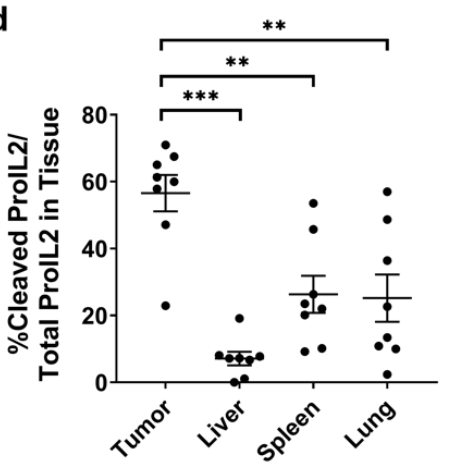

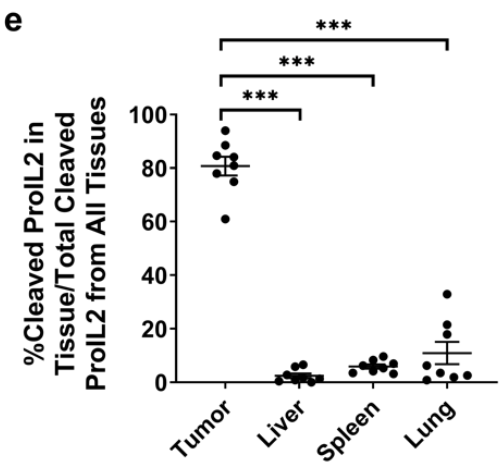


a

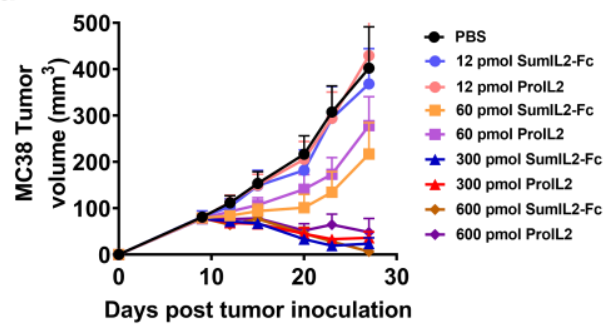

b

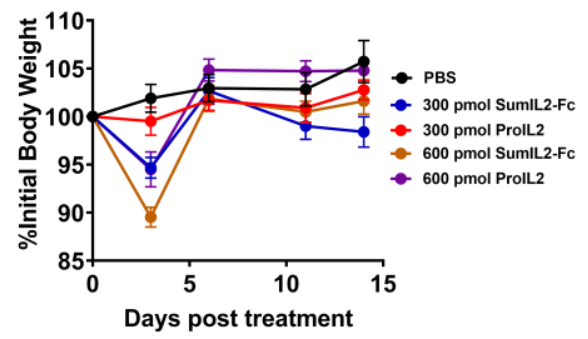

C

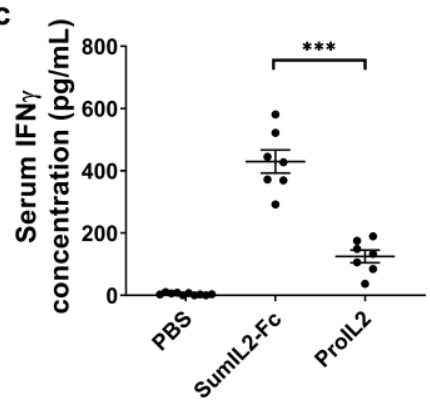

f

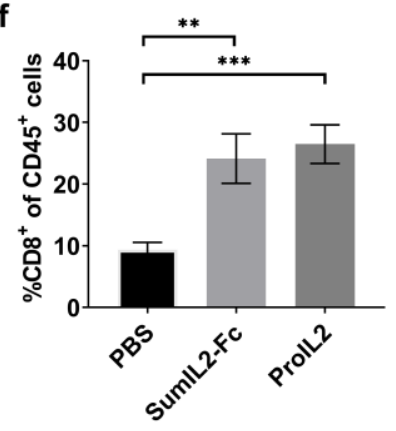

d

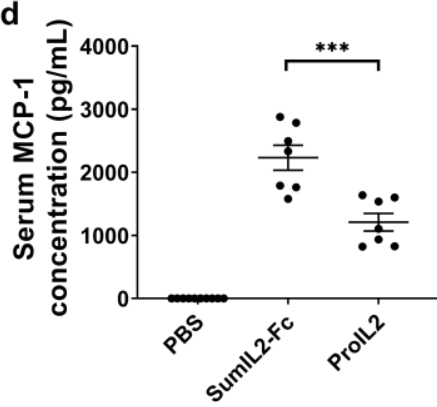

g

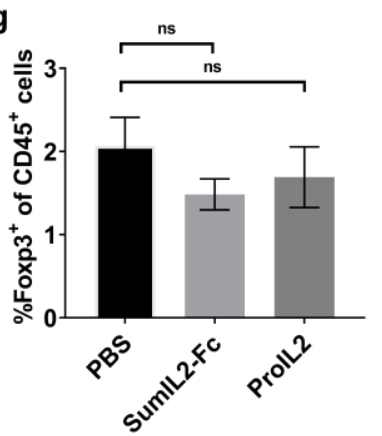

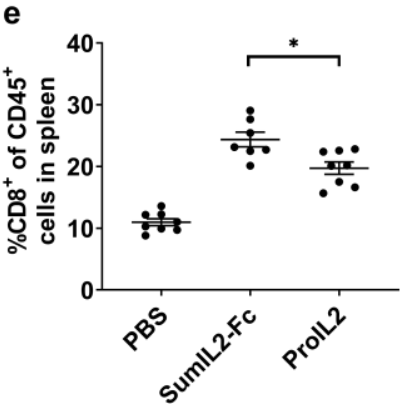

h

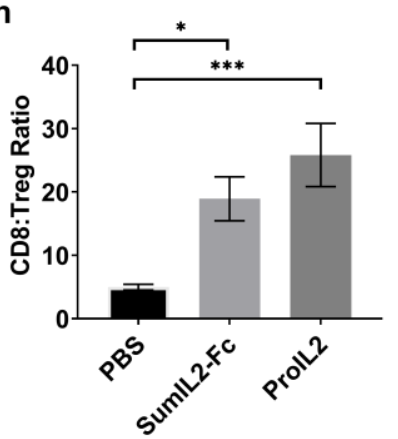


a

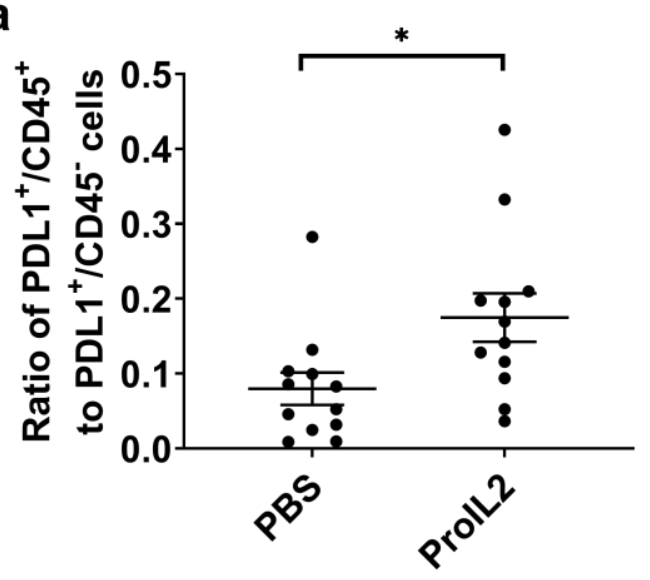

C

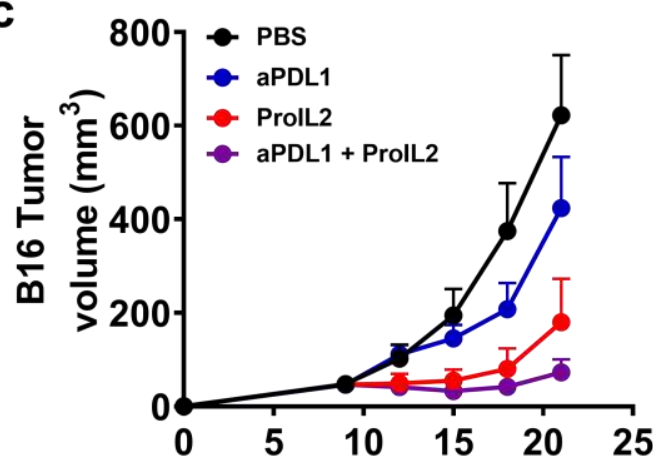

Days post tumor inoculation b
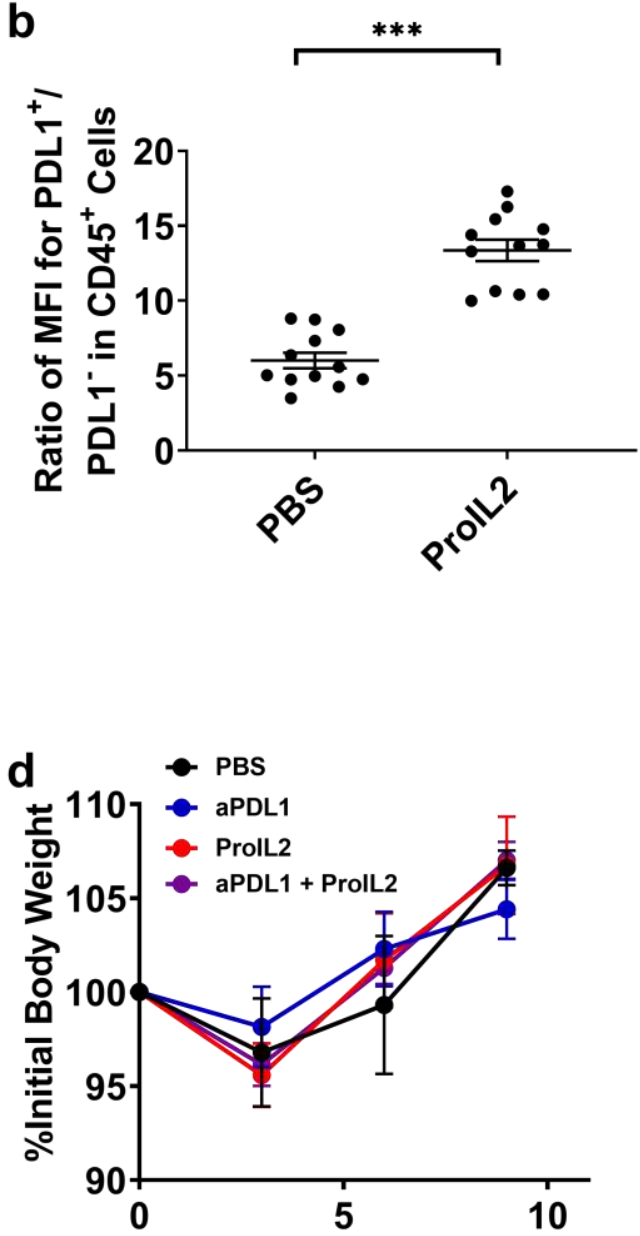

Days post tumor inoculation 
a

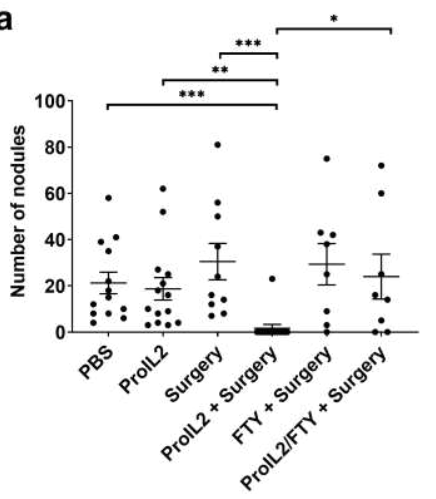

b

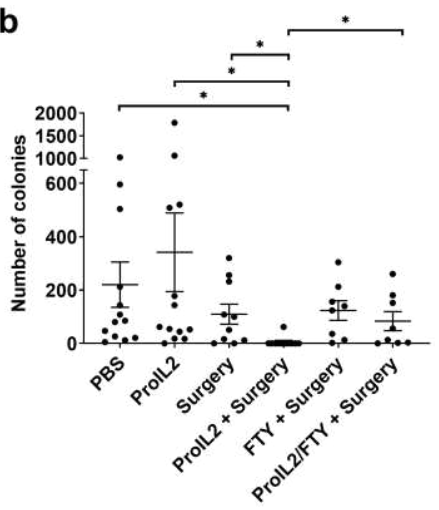

C

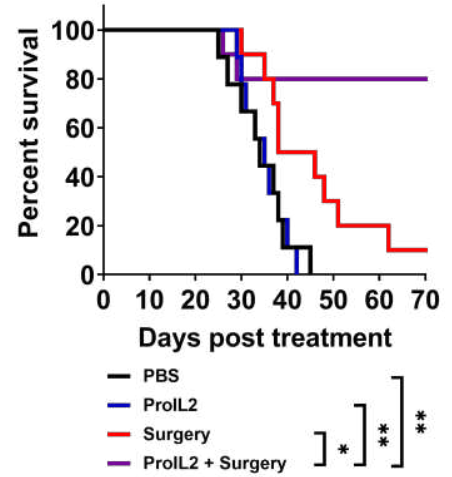




\section{Figures}

a

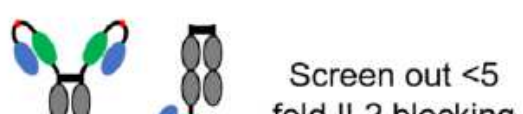
fold IL2 blocking activity or no 88 8 MMP cleavage
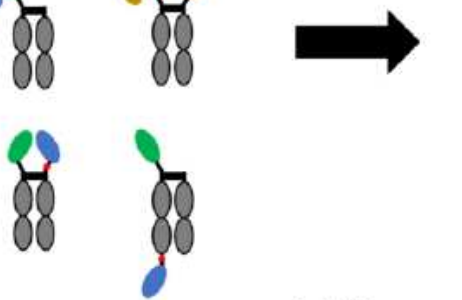

In Vitro Screening

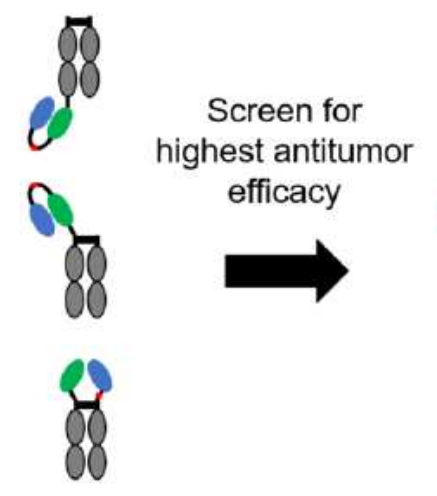

In Vivo Screening
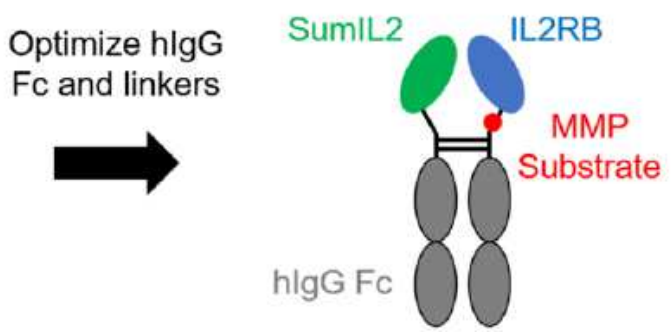

Final ProlL2

b

\begin{tabular}{|c|c|c|c|c|c|}
\hline Arm 1 & Arm 2 & IC50 & Fold Block of IL2 Activity & $\%$ Cleavage & $\%$ Tumor Reduction \\
\hline SumlL2-Fc & $\mathrm{Fc}$ & $43.9 \pm 4.5$ & N/A & N/A & $93.8 \pm 2.8$ \\
\hline IL2RB-Fc & SumlL2-Fc & $1072.6 \pm 115.8$ & 24.4 & 0 & $4.5 \pm 17.0$ \\
\hline IL2R $\beta-M M P-F c$ & SumlL2-Fc & $371.6 \pm 50.0$ & 8.5 & 66.4 & $89.8 \pm 3.0$ \\
\hline IL2Rß-MMP-IL2R $\alpha-F c$ & WTIL2-Fc & $360.7 \pm 24.2$ & 8.2 & 52.8 & $75.5 \pm 11.5$ \\
\hline IL2R $\beta^{*}-M M P-S u m \| 2-F c$ & Fc & $347.4 \pm 17.9$ & 7.9 & 29.1 & $56.6 \pm 10.2$ \\
\hline IL2Rß-MMP-SumIL2-Fc & $\mathrm{Fc}$ & $846.1 \pm 40.0$ & 19.3 & 14 & $54.3 \pm 11.7$ \\
\hline Fc-SumIL2-MMP-IL2R $\beta$ & Fc & $1301.0 \pm 229.5$ & 29.5 & 15.8 & $24.5 \pm 19.8$ \\
\hline IL2Rß-MMP-IL2R $\alpha-W T I L 2-F c$ & $\mathrm{Fc}$ & $4837.7 \pm 1032.6$ & 110.0 & 28.6 & $21.8 \pm 25.1$ \\
\hline
\end{tabular}

\section{Figure 1}

Engineering ProlL2 Design. a Pipeline for designing and screening the most optimal ProlL2 designs. ProlL2 designs were screened using HEK-BlueTM IL-2 reporter cell assay, MMP cleavage assay for 4 hours, and tumor inoculation and growth measurement as described in the methods. $b$ Multiple designs of ProlL2 that passed the in vitro screening stage were tested. The Fc arms, listed from $\mathrm{N}$ terminus to $\mathrm{C}$ terminus in each entry, of each proposed design are shown. HEKBlueTM IL-2 Reporter cell assay was used to determine the IC50 in nM of functional activity of each ProlL2 design. Fold block of IL2 activity was measured by dividing the IC50 of each ProlL2 design to that of SumIL2-Fc. Each ProlL2 design was cleaved by MMPs and run on SDS-PAGE, where the amount of cleaved vs uncleaved ProlL2 product was quantified. MC38 s.c. tumor bearing mice were injected i.p. with PBS and an equimolar dose of the listed ProlL2 design 9 days after tumor inoculation. 12 days after treatment, tumor volumes of PBS treated and ProlL2 treated mice were measured, and the decrease in tumor volume in ProlL2 treated mice on this day was quantified. Abbreviations: IL2R $\beta=$ IL-2 Receptor Beta, IL2R $\beta^{\star}=$ Truncated version of IL-2 Receptor 
Beta, MMP = 10-mer cleavable MMP substrate, IL2Ra = IL2 Receptor Alpha, WTIL2 = Wild-type IL-2. Data represent the mean \pm s.e.m of replicates.

a

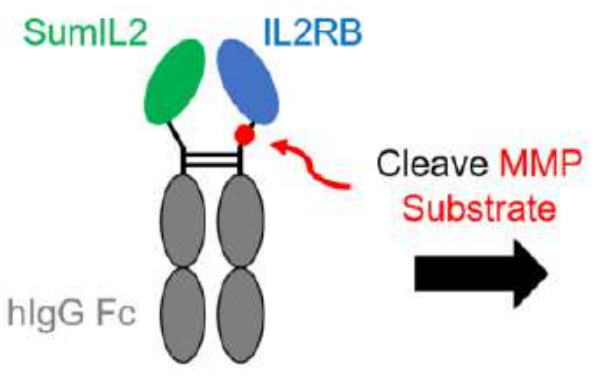

ProlL2

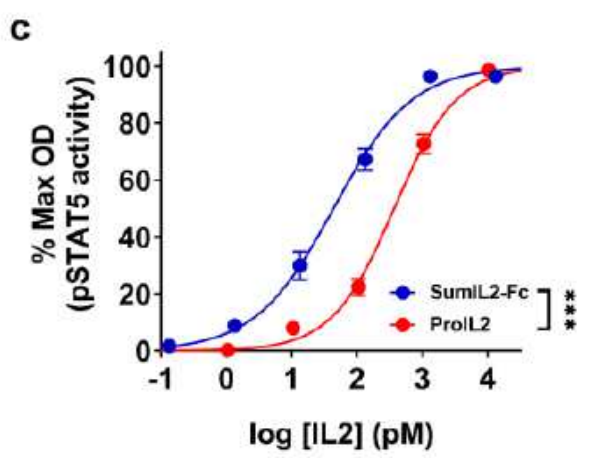

d

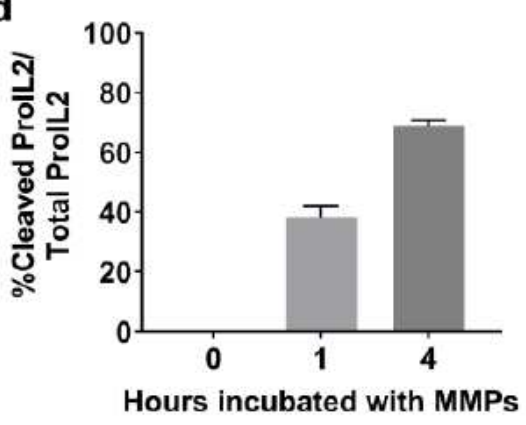

b

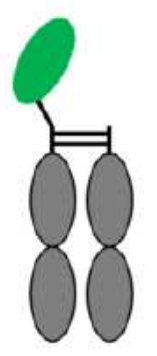

SumIL2-Fc

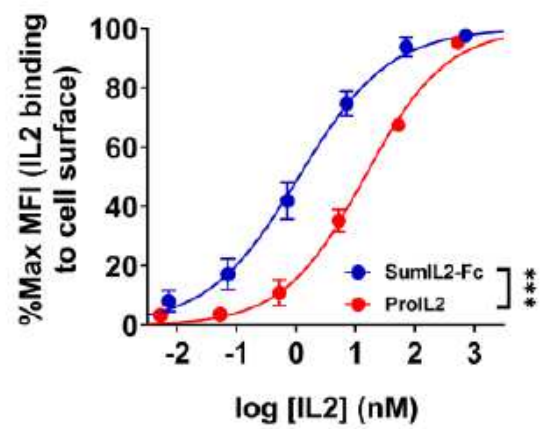

e

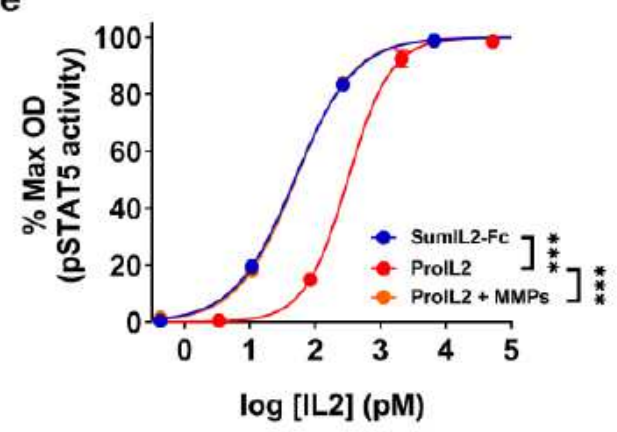

\section{Figure 2}

ProlL2 effectively masks IL-2 activity and is cleaved by MMPs in vitro. a Schematic of ProlL2 depicting that IL2RB binds and blocks IL-2 activity prior to cleavage (left) and activates into SumIL2-Fc upon MMP cleavage (right). b Binding of SumIL2-Fc and ProlL2 at different doses to HEK-BlueTM IL-2 Reporter cells was assessed via flow cytometry ( $\mathrm{n}=3$ experiments, 3 replicates each). c Functional activity of SumIL2-Fc and ProlL2 was assessed by using HEK-BlueTM IL-2 Reporter cell assay ( $\mathrm{n}=3$ experiments, 3 replicates each). $d$ Protease activation of ProlL2. ProlL2 was incubated with human MMP2, MMP9, and MMP14 for 1 or 4 hours, then run on non-reducing SDS-PAGE, where gel band intensity was subsequently quantified ( $n=3$ experiments). e Functional activity of SumIL2-Fc, ProlL2, and ProlL2 that was incubated with human MMP2, MMP9, and MMP14 for 4 hours was assessed by using HEK-BlueTM IL-2 Reporter cell assay ( $n=1$ of 2 experiments, 3 replicates each). Data represent the mean \pm s.e.m of replicates. 
a

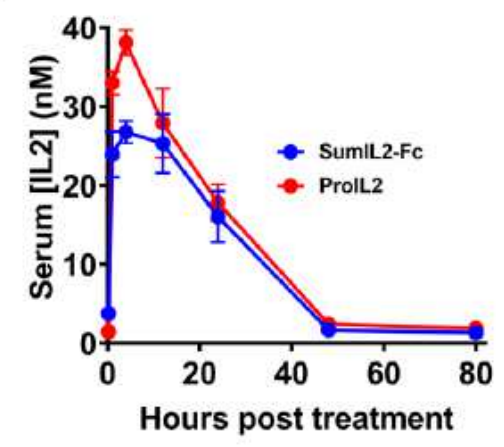

b

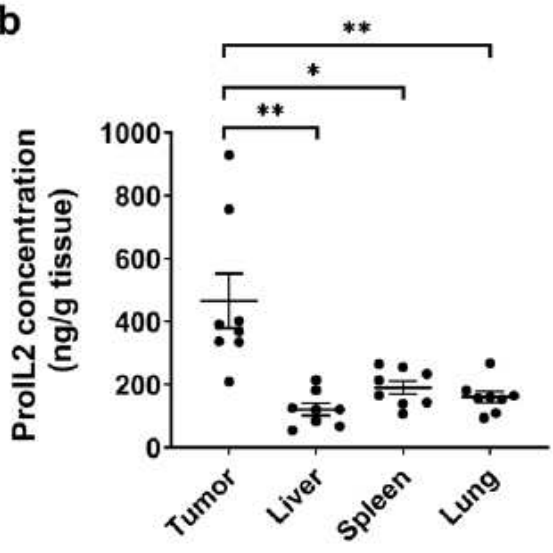

C

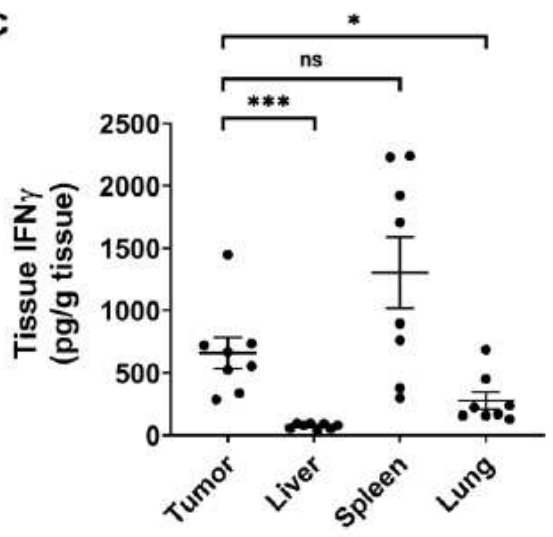

d

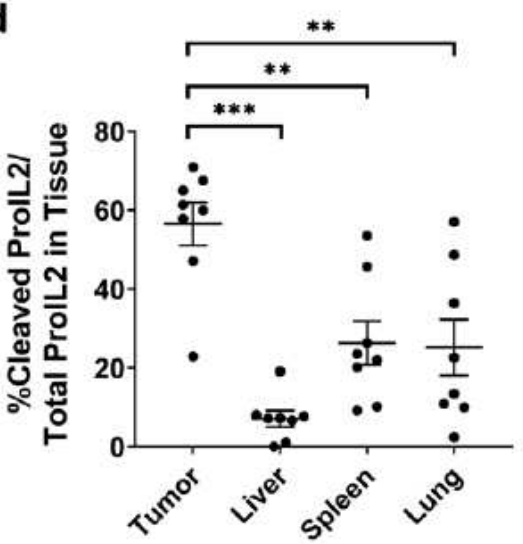

e

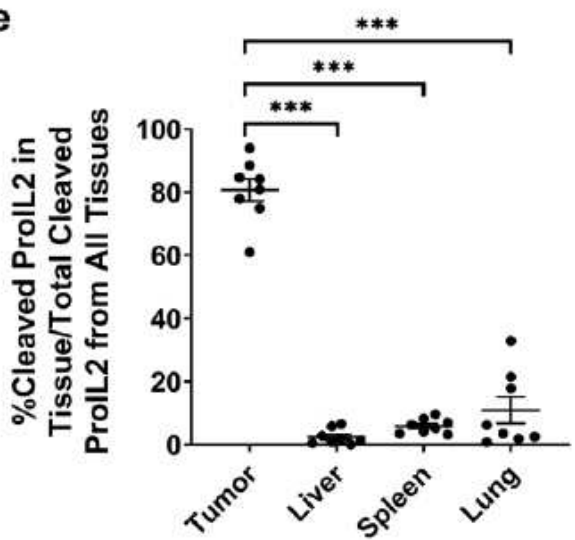

Figure 3

ProlL2 preferentially localizes to and cleaves effectively in tumors. a Equimolar doses of SumIL2-Fc (20 $\mu \mathrm{g})$ or ProlL2 $(30 \mu \mathrm{g})$ was injected i.p. into CT26 s.c. tumor bearing mice, and serum was collected and isolated at multiple time points. hIgG ELISA was used to quantify the amount of SumIL2-Fc or ProlL2 in the serum ( $n=2$ experiments, 4 replicates each). b-e $20 \mu \mathrm{g}$ ProlL2 was injected i.p. into CT26 s.c. tumor bearing mice, and the labeled tissues were collected and homogenized 24 hours after treatment $(n=2$ experiments, 4 replicates each). b hlgG ELISA was used to quantify the amount ProlL2 in each homogenate, normalized by total tissue weight. The quantity within each mouse were then normalized to the amount of ProlL2 in that mouse's tumor. c Cytometric Bead Array was used to quantify the amount of IFN囚 in each tissue homogenate. d-e hlgG immunoprecipitation of equivalent weights of tissue homogenate was performed with Protein A binding beads, and then run on Western Blot with hlgG binding antibody. $\mathrm{d}$ Relative gel band intensity per lane was quantified. e Western Blot relative gel band intensities were quantified, and within each mouse, the total percentage of cleaved ProlL2 between each tissue was compared and quantified. Data represent the mean \pm s.e.m of replicates. 
a

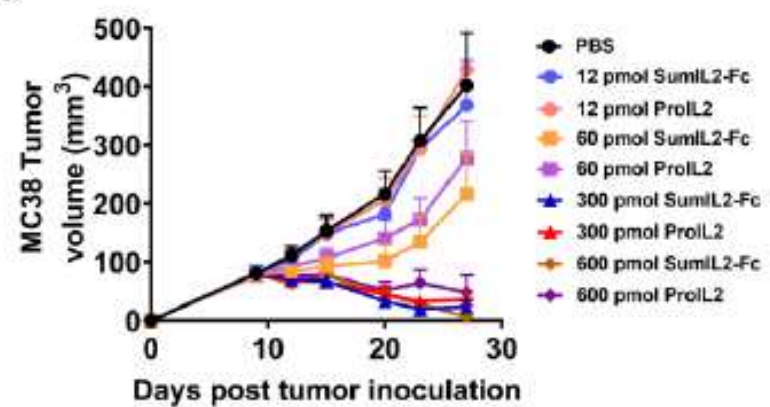

b

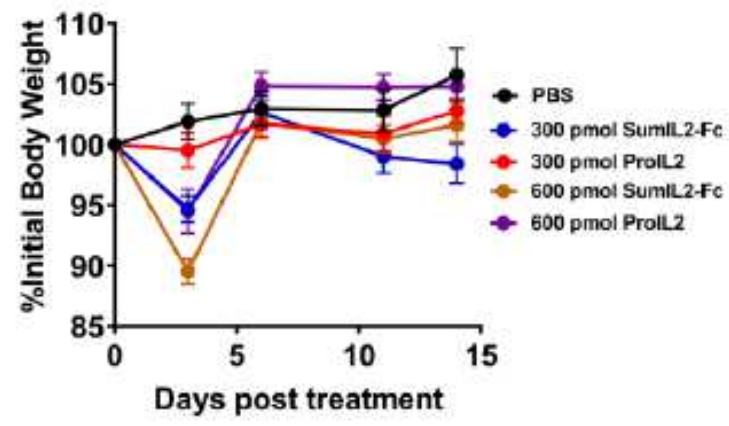

c

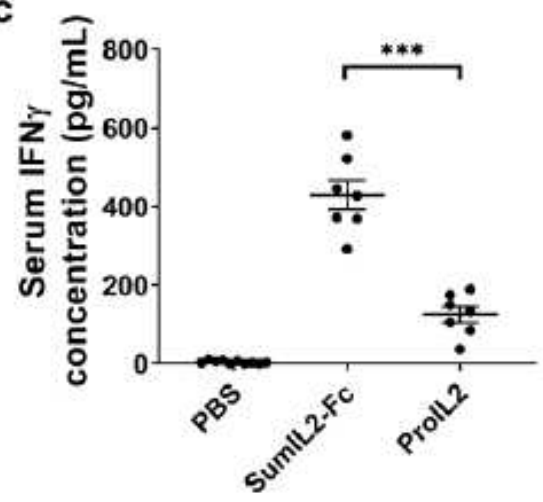

f

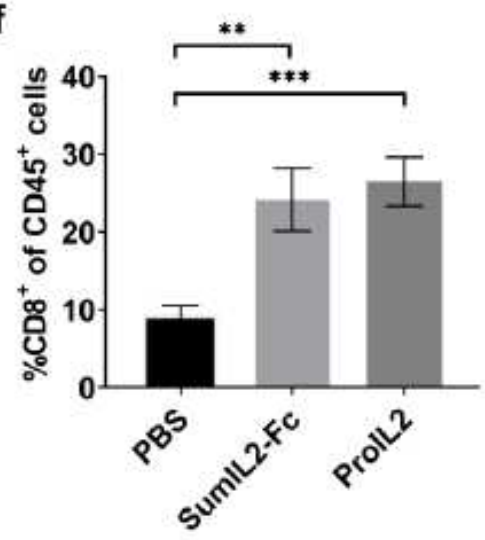

d

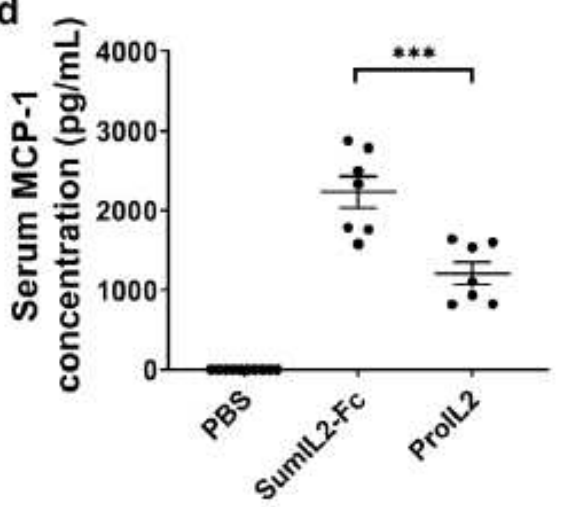

g

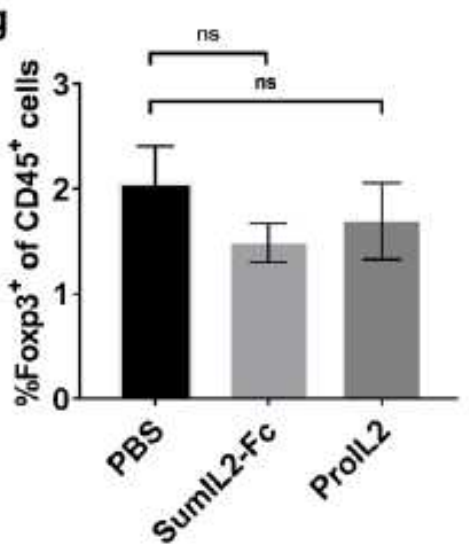

e

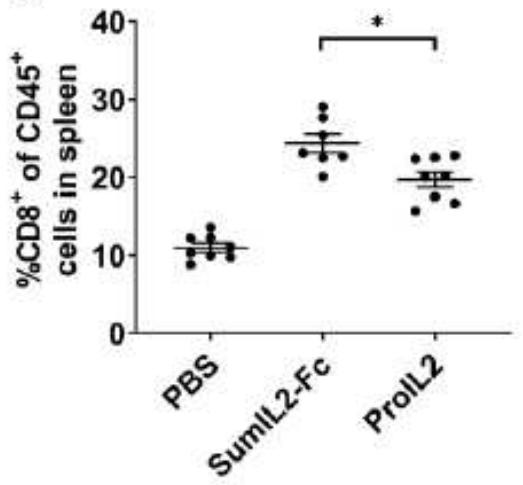

h

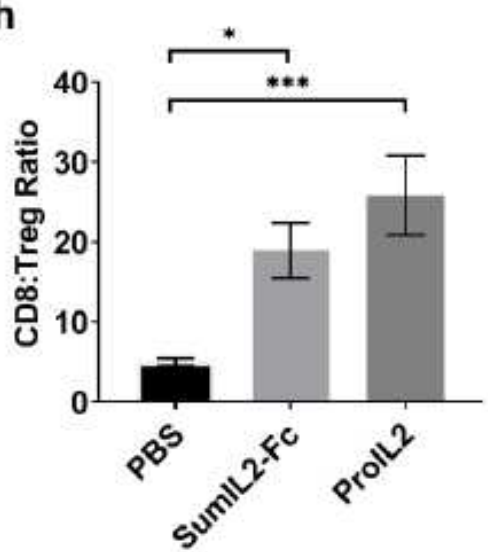

Figure 4

In vivo assessment of ProlL2. a-b MC38 s.c. tumor bearing mice were injected i.p. with one dose of the labeled treatment on day 9 post tumor inoculation; tumor growth and body weight were measured $(n=2$ experiments, 3-4 replicates each). c-e B16 s.c. tumor bearing mice were injected i.p. with PBS, SumIL2-Fc or ProlL2 (120 pmol) one time on day 9 post tumor inoculation ( $n=2$ experiments, 3-4 replicates each). Serum was collected and isolated from mice 24 hours post treatment, and Cytometric Bead Array was used to quantify the amount of serum IFN囚 or MCP-1. e 72 hours after treatment, mice were euthanized and spleens were extracted and resuspended as single cells. Flow cytometry was used to quantify the 
amount of CD8 T cells in each respective group. f-h B16 s.c. tumor bearing mice were injected i.p. with PBS, SumIL2-Fc or ProlL2 (300 pmol) one time on day 9 post tumor inoculation ( $\mathrm{n}=4$ experiments, 4 replicates each). 72 hours after treatment, mice were euthanized and tumors were extracted, digested in collagenase/DNAse, and resuspended as single cells. Flow cytometry was used to quantify the amount of CD8 T cells, amount of Tregs, and CD8:Treg ratio in each respective group. Data represent the mean \pm s.e.m. of replicates.

a

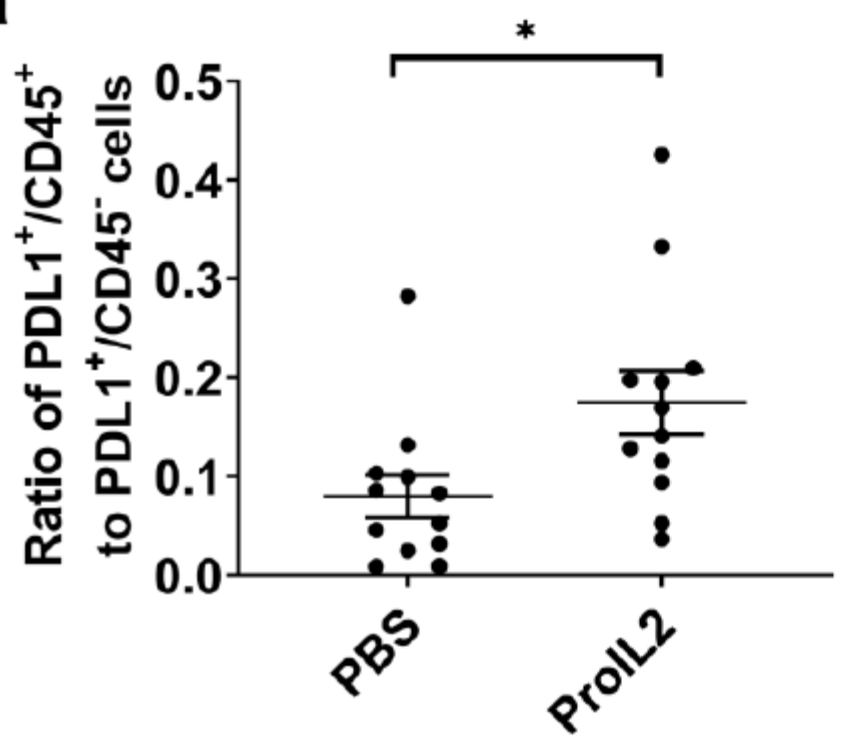

C

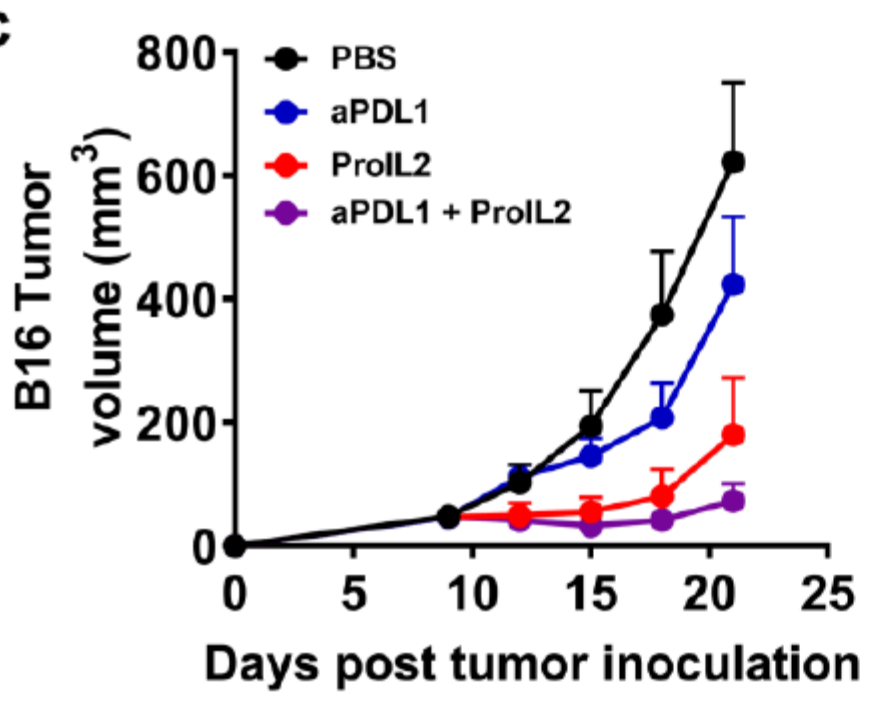

b
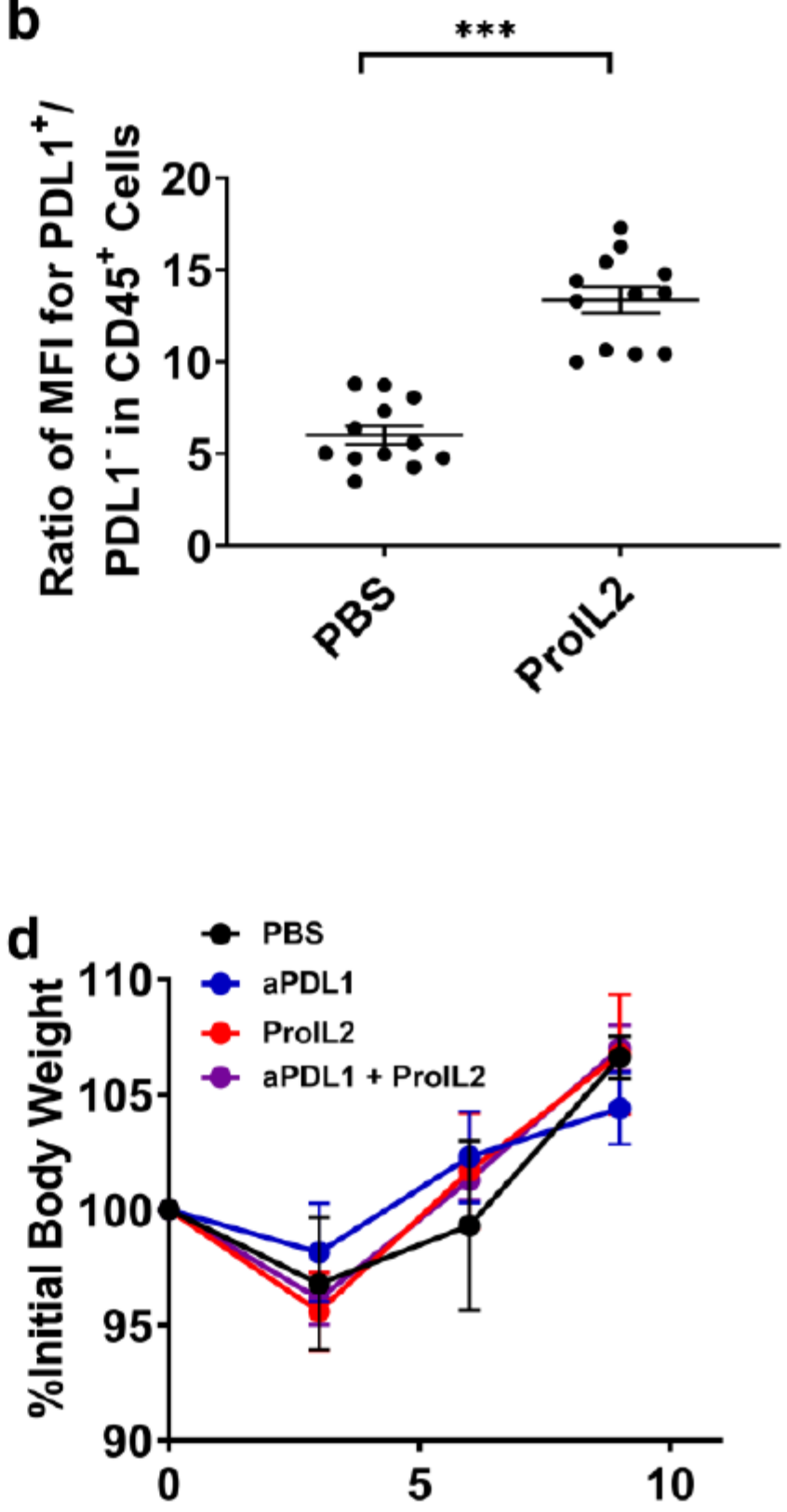

Days post tumor inoculation

Figure 5

ProlL2 can overcome advanced tumor resistance to ICB. a-b B16 s.c. tumor bearing mice were injected i.p. with PBS or ProlL2 (300 pmol) one time on day 9 post tumor inoculation. 72 hours after treatment, mice were euthanized and tumors were extracted, digested in collagenase/DNAse, and resuspended as single 
cells. Flow cytometry was used to quantify the amount of PDL1+ and CD45+ cells. In CD45+ cells, the MFI of PDL1+ and PDL1- cells were compared ( $n=3$ experiments, 4 replicates each). c-d B16 s.c. tumor bearing mice were injected i.p. with PBS, anti-PDL1 (200 $\mu \mathrm{g}$ each), ProlL2 (300 pmol), or anti-PDL1 and ProlL2 one time on day 9 post tumor inoculation; tumor growth and body weight were measured $(n=1$ of 2 experiments, 3-4 replicates each). Data represent the mean \pm s.e.m. of replicates.
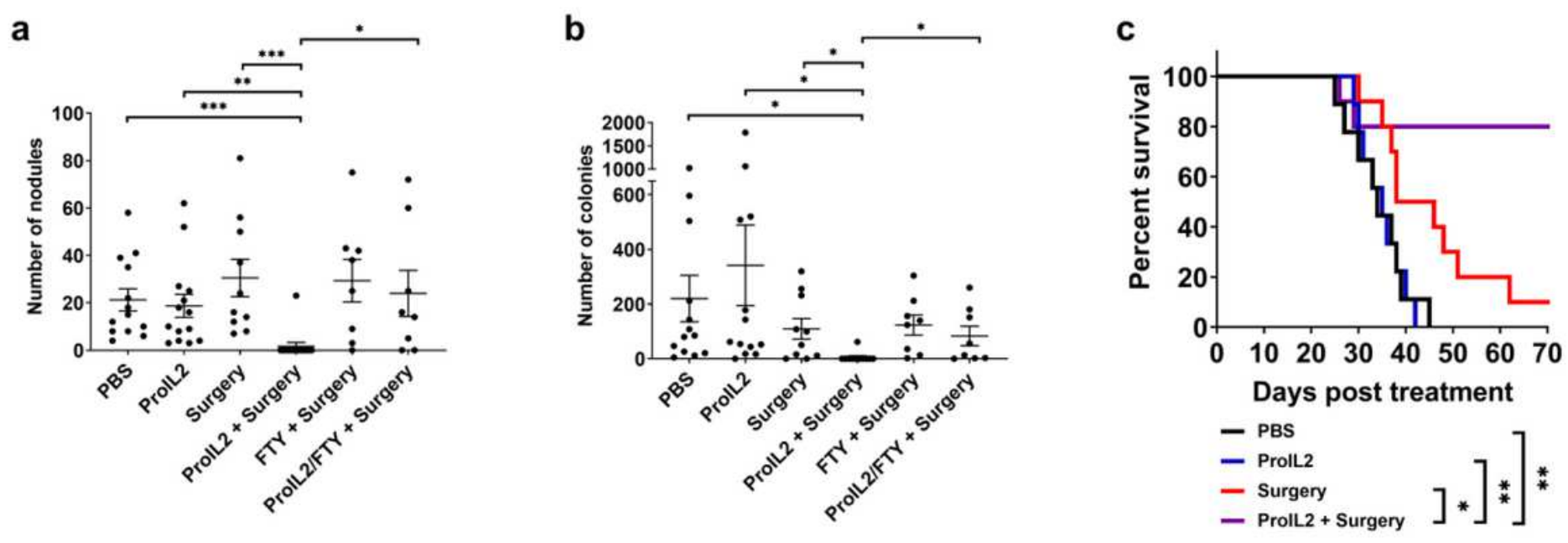

Figure 6

Preoperative ProlL2 combined with surgery can eliminate metastases through an abscopal effect. 4T1 s.c. tumor bearing mice were injected i.p. with PBS or ProlL2 $(300 \mathrm{pmol})$ on day 9 and 12 post tumor inoculation. Mice treated with FTY720 were i.p. treated with $10 \mu \mathrm{g}$ every other day for 14 days starting on day 8 post tumor inoculation. On day 14 post tumor inoculation, the listed mice had their tumors surgically removed. a 25 days post initial treatment, mice were euthanized and metastatic nodules in the thoracic cavity were counted. b 25 days post initial treatment, mice were euthanized and lungs were extracted, digested in collagenase/DNAse, and resuspended as single cells. Resuspended cells were plated at a 1:400 ratio with 6-thioguanine selection medium, and the number of colonies were counted 6 days post seeding ( $n=2$ experiments, 2-5 replicates each). c Mice were analyzed for survival starting the day after the first i.p. injection treatment ( $n=2$ experiments, 5 replicates each). Data represent the mean \pm s.e.m. of replicates.

\section{Supplementary Files}

This is a list of supplementary files associated with this preprint. Click to download.

- CXUsersIdq5835DesktopFuEHSupplementaryFigures101220.pdf 